\title{
Controlling excited-state contributions with distillation in lattice QCD calculations of nucleon isovector charges $g_{S}^{u-d}, g_{A}^{u-d}, g_{T}^{u-d}$
}

\author{
Colin Egerer* \\ Department of Physics, William and Mary, Williamsburg, Virginia 23185, USA \\ David Richards and Frank Winter \\ Thomas Jefferson National Accelerator Facility, Newport News, Virginia 23606, USA
}

(Received 26 October 2018; published 19 February 2019)

\begin{abstract}
We investigate the application of the distillation smearing approach, and the use of the variational method with an extended basis of operators facilitated by this approach, on the calculation of the nucleon isovector charges $g_{S}^{u-d}, g_{A}^{u-d}$, and $g_{T}^{u-d}$. We find that the better sampling of the lattice enabled through the use of distillation yields a substantial reduction in the statistical uncertainty in comparison with the use of alternative smearing methods, and furthermore, appears to offer better control over the contribution of excited states compared to use of a single, local interpolating operator. The additional benefit arising through the use of the variational method in the distillation approach is less dramatic, but nevertheless significant given that it requires no additional Dirac inversions. Dependence of our results on the rank of the distillation is space is also explored, and found to be mild.
\end{abstract}

DOI: $10.1103 /$ PhysRevD.99.034506

\section{INTRODUCTION}

The past decade has seen tremendous improvement in the ability of Lattice QCD (LQCD) calculations to provide results that can confront experiment. However, lattice computations of some key quantities remain at odds with experimental determinations, including the momentum fraction carried by partons in the nucleon, and, notably, the axial-vector charge, $g_{A}^{u-d}$, of the nucleon. These discrepancies are often attributed to finite-volume effects, and to the contribution of excited states to ground-state matrix elements. The calculation of the axial-vector charge has been a particular focus within the lattice community, and a dedicated effort to resolve these discrepancies has demonstrated success [1], using a method to control excited states inspired by the Feynman-Hellman theorem. In this paper we explore a different means of controlling excited states, through the use of a novel smearing method, "distillation" [2], and the variational method for the case of the nucleon charges $g_{A}^{u-d}, g_{S}^{u-d}$ and $g_{T}^{u-d}$.

The matrix element of the flavor nonsinglet axial-vector current $A_{\mu}^{a}=\bar{\psi} \gamma_{\mu} \gamma_{5} \frac{\tau^{a}}{2} \psi$, where $\psi$ is the isospin doublet of $u, d$ quark fields and $\tau$ the Pauli-spin matrices acting in

\footnotetext{
*cpegerer@email.wm.edu
}

Published by the American Physical Society under the terms of the Creative Commons Attribution 4.0 International license. Further distribution of this work must maintain attribution to the author(s) and the published article's title, journal citation, and DOI. Funded by SCOAP . isospin space, between nucleons of momenta $p$ and $p^{\prime}$ can be expressed in terms of the axial and the induced pseudoscalar and tensor form factors

$$
\begin{gathered}
\left\langle N\left(p^{\prime}, s^{\prime}\right)\left|A_{\mu}^{3}\right| N(p, s)\right\rangle=\bar{u}_{N}\left(p^{\prime}, s^{\prime}\right)\left[\gamma_{\mu} \gamma_{5} G_{A}\left(q^{2}\right)\right. \\
\left.+\frac{q_{\mu}}{2 M_{N}} \gamma_{5} G_{P}\left(q^{2}\right)+i \frac{\sigma^{\mu \nu} q_{\nu}}{2 M_{N}} \gamma_{5} G_{T}\left(q^{2}\right)\right] \frac{\tau^{3}}{2} u_{N}(p, s)
\end{gathered}
$$

where $q_{\mu}=p_{\mu}^{\prime}-p_{\mu}$ the momentum transfer. At zeromomentum transfer, the contribution of the pseudoscalar and tensor form factors to the matrix element of Eq. (1) vanish, and we are left with the definition of the isovector axial charge of the nucleon $g_{A}^{u-d}=G_{A}(0)$. The axial-vector charge $g_{A}$ quantifies the $n \rightarrow p e^{-} \bar{\nu}_{e}$ coupling, the degree of low-energy chiral symmetry breaking in QCD, and even the difference between the $u$ and $d$ quark contributions to the proton spin $g_{A}=\Delta u-\Delta d$. The breadth of different phenomena dependent on a knowledge of $g_{A}$ highlights the need for precise agreement between theoretical and experimental determinations. Moreover, the straightforward definition of $g_{A}$ serves as a useful proving ground for any new lattice algorithm purporting to calculate meaningful quantities in QCD.

Precise neutron $\beta$ decay experiments have measured the nucleon isovector axial charge $g_{A}^{u-d}=1.2724 \pm 0.0023$ [3]. Yet, historically, the bulk of lattice calculations have systematically underdetermined $g_{A}^{u-d}$ by roughly $10 \%-15 \%$. This has led to intense discussion in the community on the 
role of finite-volume effects, the lack of chiral symmetry in most lattice formulations of QCD, the influence of heavy quark flavors, the use of local currents with large discretization effects, and excited-state contamination might have on the calculation of $g_{A}^{u-d}$ and nucleon matrix elements in general. Calculations have been performed using the highly improved staggered quark [4], domain wall [5] and overlap [6] fermion actions which, despite the restoration of chiral symmetry, present challenges, namely, identifying quark flavors and the numerical costs compared to Wilson-type actions, respectively. Calculations have even been performed with $\mathcal{O}\left(a^{2}\right)$ improved currents with novel noise reduction techniques [7,8], and others still with $2+1+1$ flavor QCD accounting for several excited states in requisite fits $[9,10]$. Substantial effort to calculate nucleon charges has also been made in the framework of twisted mass QCD (e.g., [11,12]), in which exceptional configurations are removed and automatic $\mathcal{O}(a)$ improvement is achieved at maximal twist. In all such cases the calculated value of $g_{A}^{u-d}$ differs from experiment by $\sim 5 \%-10 \%$. Clearly standard techniques of calculating and subsequently fitting two- and three-point correlation functions to extract $g_{A}^{u-d}$ routinely come up short.

Recent work by [1] employs a methodology inspired by the Feynman-Hellman theorem to control excited-state effects by summing over all current insertion times, engendering extrapolation in a single Euclidean temporal variable rather than two-agreement was found to within $1 \%$ of experiment. We remark that a few 2-flavor lattice QCD calculations employing the Wilson plaquette/clover fermion actions have been performed for $g_{A}^{u-d}$ whose results are within $\sim 1 \%$ of the experimental determination $[13,14]$. These studies calculated $g_{A}^{u-d}$ for source-sink separations greater than $1 \mathrm{fm}$. Furthermore it was found $g_{A}^{u-d}$ has a strong dependence on the spatial lattice volume $V_{3}$. Taken together, suggesting the largest contributors to uncertainty in $g_{A}^{u-d}$ lie in small lattice volumes and excited-state contamination.

In this paper, we investigate a means of overcoming one of the dominant systematic uncertainties in the calculation of nucleon charges, namely that arising from our inability to isolate the ground-state nucleon from its excitations. The variational method, an algorithm to improve overlap onto a desired state, was applied in [15-17] to a small basis of Jacobi-smeared nucleon interpolators of the form $\chi(x)=$ $\epsilon^{a b c}\left[u^{a \top}(x) C \gamma_{5} d^{b}(x)\right] u^{c}(x)$, where it was found variationally improved interpolators can reduce excited-state effects. We will show that an amalgam of a suitable basis of interpolating operators, the use of the variational method with that basis, and an efficient means of computing the needed correlation functions through the application of distillation, affords a powerful and computationally efficient method of taming excited-state contributions to those charges. Furthermore, we will show that distillation alone, enabling a momentum projection to be performed at each time slice in the two- and three-point functions, provides a significant increase in the statistical precision while being an effective smearing operator with only a single interpolating operator. This allows the reliable extraction of matrix elements at earlier source-sink separations when the nucleon signal-to-noise ratio is exponentially better. As lattice calculations proceed to ever more complicated quantities, exemplified by quasi-PDFs and pseudo-PDFs, the need for tamed excited-state effects is paramount.

In this work, we abstain from calculating and presenting renormalized isovector charges of the ground-state nucleon, and from performing continuum, finite-volume, and chiral extrapolations. Throughout this work we consider forwardscattering matrix elements of nucleons at rest. Although much of our motivating comments have centered around $g_{A}^{u-d}$, we extend our technique to include the scalar $g_{S}^{u-d}$ and tensor $g_{T}^{u-d}$ charges of the nucleon, whose precise determination will constrain BSM searches at the TeV scale and dark matter direct-detection searches. A future study will explore forward-scattering matrix elements of moving states, and generalizations to scalar, axial, and tensor nucleon form factors.

This paper is organized as follows. In Sec. II we discuss excited-state contamination and present the computational methodology employed throughout this work. In particular, we review distillation as a low-mode approximation of the more standard Jacobi and Wuppertal smearing algorithms, and discuss the variational method as a means of improving overlap onto a desired state. Section III describes the lattice ensemble, the explicit construction of the interpolating fields, and then finishes with a discussion of the strategy used to extract the nucleon charges from our data. In Sec. IV we first feature a comparison of the nucleon effective masses obtained using a Jacobi-smeared interpolator, a single "local" distilled interpolator, and different variationally improved interpolators from distinct bases of distilled interpolators. We then proceed to present determinations of effective masses of the nucleon via fits to our data and ultimately our extracted charges. We then conclude with a discussion of our results, a cost-benefit analysis of standard smearing techniques and distillation, implications for assorted studies in nucleon structure, and directions for future research.

\section{COMPUTATIONAL METHODOLOGY}

We begin this section with some definitions we will use throughout this work. Isovector charges $g_{\Gamma}$ of the nucleon are measured experimentally through the neutron to proton transitions $\left\langle p(p, s)\left|\mathcal{O}_{\Gamma}^{u d}\right| n(p, s)\right\rangle$, where $\mathcal{O}_{\Gamma}^{u d}=\bar{u} \Gamma d$, or via proton and neutron charge differences. Imposing isospin symmetry, one can show

$$
\left\langle p(p, s)\left|\mathcal{O}_{\Gamma}^{u d}\right| n(p, s)\right\rangle=\left\langle p(p, s)\left|\mathcal{O}_{\Gamma}^{u-d}\right| p(p, s)\right\rangle
$$

where $\mathcal{O}_{\Gamma}^{u-d}=\bar{u} \Gamma u-\bar{d} \Gamma d$ is an external current. The isovector charges of the nucleon are thus defined as 


$$
\left\langle N(p, s)\left|\mathcal{O}_{\Gamma}^{u-d}\right| N(p, s)\right\rangle=g_{\Gamma}^{u-d} \bar{u}_{s}(p) \Gamma u_{s}(p)
$$

where we normalize the nucleon spinors in Euclidean space according to

$$
\sum_{s} u_{s}(p) \bar{u}_{s}(p)=-i \not{ }_{E}+m_{N}
$$

Indeed the calculation of isovector quantities is computationally less demanding than the calculation of isoscalar and flavor-diagonal quantities, in which the cancellation of disconnected quark lines in the isospin limit does not occur.

\section{A. Excited-state contamination}

The calculation of hadronic matrix elements within LQCD requires the construction of operators that attempt to interpolate a given hadronic state from the vacuum, with minimal overlap with neighboring states. As the precise wave function of any hadronic state is not known, any operator construction of a desired continuum $J^{P C}$ is merely a "best guess" and necessarily overlaps with other hadronic states of the same quantum numbers - most notably, excited states and multiparticle states. This problem is compounded with the explicit breaking of Lorentz symmetry, in which continuum operators residing in different irreducible representations (irreps) of the Lorentz group can mix under subduction to the same lattice irrep thereby increasing the number of states contributing within a given lattice irrep.

Explicitly, consider a two-point correlation function projected to zero momentum

$$
C_{2 \mathrm{pt}}(t)=\sum_{\vec{x}}\langle\mathcal{O}(\vec{x}, t) \overline{\mathcal{O}}(\overrightarrow{0}, 0)\rangle
$$

where $\mathcal{O}$ is an interpolating operator for the state of interest. Performing a spectral decomposition, this can be expressed as

$$
C_{2 \mathrm{pt}}(t)=\sum_{n} \frac{1}{2 E_{n}}|\langle 0|\mathcal{O}| n\rangle|^{2} e^{-E_{n} t},
$$

where the sum is over all states, of energy $E_{n}$, that can be created with the operator $\mathcal{O}$. In order to extract reliably the ground-state mass $M_{0} \equiv E_{0}$, one can study the large time behavior of the two-point correlator, wherein contributions of excited states are negligible, or make a judicial choice of interpolating operator such that the overlap factors $Z_{n}=\langle 0|\mathcal{O}| n\rangle$, for states $n>0$, are greatly suppressed relative to $\langle 0|\mathcal{O}| n=0\rangle$, and thereby determine $E_{0}$ at small temporal separations. Given that lattice calculations of baryon properties are constrained by an exponentially increasing noise-to-signal ratio with increasing Euclidean time, the latter approach is far preferable. This issue is further compounded when considering matrix elements of an external current $\mathcal{J}$, where the suppression of excitedstates is needed both between the source interpolator and the current, and the current and the sink interpolator. Thus there is strong encouragement to develop operators that couple predominantly to the ground state, and only weakly to the excited states.

\section{B. Smearing}

Interpolating fields constructed of pointlike quark and gluonic fields couple to hadronic states at all energy scales. "Smearing" is a well-established technique to increase the overlap of interpolators with the low-lying states of the spectrum (i.e., confinement scale physics), and to reduce the contribution of the high-energy modes to correlation functions, through the use of spatially extended operators of hadronic size. Specifically, we can replace the quark fields $\psi(\vec{x}, t)$ occurring in the path integral with spatially extended quark fields

$$
\tilde{\psi}(\vec{x}, t)=\sum_{\vec{y}} S[U](\vec{x}, \vec{y}) \psi(\vec{y}, t),
$$

where $S[U](\vec{x}, \vec{y})$ is a gauge-covariant scalar smearing kernel that is functionally dependent on the underlying gauge fields $U$ on some time slice $t$.

A frequently utilized smearing operator is $J_{\sigma, n_{\sigma}}(t)=$ $\left(1+\frac{\sigma \nabla^{2}(t)}{n_{\sigma}}\right)^{n_{\sigma}}$ defining the Jacobi-smearing method [18], where $\nabla^{2}$ is a gauge-covariant discretization of the Laplacian, $\sigma$ is the smearing "width," and $n_{\sigma}$ represents the number of applications of the Laplacian. In the large $n_{\sigma}$ limit,

$$
\tilde{\psi}(\vec{x}, t)=\lim _{n_{\sigma} \rightarrow \infty} J_{\sigma, n_{\sigma}}(t) \psi(\vec{x}, t) \rightarrow e^{\sigma \nabla^{2}(t)} \psi(\vec{x}, t)
$$

thus approaching a Gaussian of width $\sigma$, characteristic of the size of a hadron.

\section{Distillation and operator construction}

Distillation[2] is a low-rank approximation to a gaugecovariant smearing kernel. Specializing to the case of the Laplacian, we begin by seeking solutions to $-\nabla^{2}(t) \xi^{k}(t)=$ $\lambda^{k}(t) \xi^{k}(t)$. Ordering solutions by the magnitude of the eigenvalues $\lambda^{k}(t)$, the distillation operator is constructed as the outer product of two eigenvectors on a given time slice

$$
\square(\vec{x}, \vec{y} ; t)_{a b}=\sum_{k=1}^{N} \xi_{a}^{(k)}(\vec{x}, t) \xi_{b}^{(k) \dagger}(\vec{y}, t),
$$

where the color indices $\{a, b\}$ are made explicit. The distillation operator $\square$ is then applied to each quark or antiquark field both at the source and at the sink.

Distillation affords several advantages over Jacobi-like smearing methods. Distillation, firstly, factorizes the construction of the interpolating or current operators from quark propagation. Secondly, distillation allows operators 
not only at the sink location but also at the source to incorporate more elaborate spatial structure, and in particular derivatives, without additional inversions of the Dirac operator. Finally, distillation enables momentum projection to be performed both at the source and sink time slices, thus providing a more thorough sampling of a given lattice configuration. In this case, Eq. (3) becomes

$$
C_{2 \mathrm{pt}}(t)=\sum_{\vec{x}, \vec{y}}\langle\mathcal{O}(\vec{x}, t) \overline{\mathcal{O}}(\vec{y}, 0)\rangle .
$$

These features have been key to the precise determination of the many energy levels in the QCD spectrum needed for the study of resonances in lattice calculations. However, these features are also of advantage in studies of hadron structure, by facilitating more varied interpolator constructions, and by enabling momentum projection at each time slice in a correlation function.

Specializing to the case of baryons, we will construct our interpolating operators $\mathcal{O}_{i}$ following the procedure of Refs. [19,20]

$$
\mathcal{O}_{i}(t) \propto \epsilon^{a b c} \mathcal{S}_{i}^{\alpha \beta \gamma}\left(\mathcal{D}_{1} \square u\right)_{a}^{\alpha}\left(\mathcal{D}_{2} \square d\right)_{b}^{\beta}\left(\mathcal{D}_{3} \square u\right)_{c}^{\gamma}(t),
$$

where $\mathcal{D}_{1,2,3}$ are spatial operators constructed from covariant derivatives, introduced to probe the radial/angular structure of the nucleon wave function, and $S^{\alpha \beta \gamma}$ are subduction matrices that project a state of definite continuum spin into irreps of the hypercubic lattice (explicit construction given in Sec. III B). The building blocks of the two-point and three-point correlation functions employing these interpolating operators are

(i) solution vectors

$$
S_{\alpha \beta}^{(k)}\left(\vec{x}, t^{\prime} ; t\right)=M_{\alpha \beta}^{-1}\left(t^{\prime}, t\right) \xi^{(k)}(t)
$$

(ii) perambulators

$$
\tau_{\alpha \beta}^{(k, l)}\left(t^{\prime}, t\right)=\xi^{(k) \dagger}\left(t^{\prime}\right) M_{\alpha \beta}^{-1}\left(t^{\prime}, t\right) \xi^{(l)}(t)
$$

(iii) elementals

$$
\begin{aligned}
\Phi_{\alpha_{1}, \alpha_{2}, \alpha_{3}}^{(i, j, k)}(t)= & \epsilon^{a b c}\left(\mathcal{D}_{1} \xi^{(i)}\right)^{a}\left(\mathcal{D}_{2} \xi^{(j)}\right)^{b} \\
& \times\left(\mathcal{D}_{3} \xi^{(k)}\right)^{c}(t) S_{\alpha_{1}, \alpha_{2}, \alpha_{3}}
\end{aligned}
$$

where it should be noted that the inversion of the lattice Dirac operator against a smeared point-source in standard techniques, is replaced in distillation by inversion of the lattice Dirac operator against the $k$ th eigenvector of time slice $t$. The principle disadvantage of the distillation method is that the number of distillation eigenvectors $N$ needed to construct a correlation function of the same resolution is expected to scale as the lattice spatial volume $V_{3}$. Since the evaluation of the Wick contractions for mesons and baryons scales as $N^{3}$ and $N^{4}$, respectively, the volume-scaling cost is potentially severe.

\section{Variational analysis}

The variational method is an often employed technique in lattice spectroscopy calculations that seeks to disentangle the contributions of eigenstates to the two-point correlation of two interpolating operators. The variational method begins by constructing a matrix of correlation functions

$$
C_{i j}(t)=\left\langle\mathcal{O}_{i}(t) \overline{\mathcal{O}}_{j}(0)\right\rangle
$$

where $\mathcal{O}_{i}$ and $\mathcal{O}_{j}$ belong to some basis $\mathcal{B}$ of appropriately constructed interpolators with identical quantum numbers. In practice, these interpolators transform with definite symmetries in quark flavor, derivative structure, and Dirac structure-where the Dirac structure of the operator is encoded in the subduction of a continuum operator into lattice irreps. The actual variational method begins by considering the system of generalized eigenvalue equations

$$
C(t) v_{\mathbf{n}}\left(t, t_{0}\right)=\lambda_{\mathbf{n}}\left(t, t_{0}\right) C\left(t_{0}\right) v_{\mathbf{n}}\left(t, t_{0}\right)
$$

with $\mathbf{n} \in\{1, \ldots, \operatorname{dim}(\mathcal{B})\}$ and $t>t_{0}$. It can be shown that at large times $t$ this system of equations is described by the "principal correlators" $\lambda_{\mathrm{n}}\left(t, t_{0}\right)=e^{-M_{n}\left(t-t_{0}\right)}$, where the trivial solution $v_{\mathrm{n}}=0$ is avoided by imposing the normalization condition $v_{m}^{\dagger} C\left(t_{0}\right) v_{n}=\delta_{m n}$. Equation (9) is solved independently for each $t>t_{0}$, with $\lambda_{\mathrm{n}}\left(t_{0}, t_{0}\right)=1$ by construction. It is possible that in the process of solving Eq. (9), what appears to be the $m$ th eigenvector on time slice $t_{m}$ is not the $m$ th eigenvector on time slice $t_{m+1}$. To remedy this, we follow the procedure of [19] by associating states on different time slices using the relative similarity between their associated eigenvectors. This is accomplished by selecting some reference time slice $t_{\text {ref }}$ and maximizing $v_{m}^{\text {ref } \dagger} C\left(t_{0}\right) v_{n}$, where $v_{m}^{\text {ref }} \equiv v_{m}\left(t_{\text {ref }}\right)$.

A fitting function

$$
\lambda_{\mathrm{n}}\left(t, t_{0}\right)=\left(1-A_{\mathrm{n}}\right) e^{-M_{\mathrm{n}}\left(t-t_{0}\right)}+A_{\mathrm{n}} e^{-M_{\mathrm{n}}^{\prime}\left(t-t_{0}\right)}
$$

is applied to the principal correlator to obtain the masses $M_{\mathrm{n}}, M_{\mathrm{n}}{ }^{\prime}$, and amplitude $A_{\mathrm{n}}$. The choice for $t_{0}$ is made by attempting to reconstruct the original correlation matrix $C_{i j}(t)$ using the masses $M_{\mathrm{n}}, M_{\mathrm{n}}{ }^{\prime}$ and the extracted overlap factors $Z_{i}^{\mathrm{n}}$ [21]. The degree of agreement for $t>t_{0}$ dictates the choice for $t_{0}$. Solving the variational method introduces a slight time dependence in the overlap factors. The overlap factors are thus evaluated on a time slice $t_{Z}>t_{0}$ that minimizes differences between original/reconstructed correlators [21]. The resulting eigenvectors $v_{\mathbf{n}}$ for each $t>t_{0}$ yield the optimal linear combination of $\mathcal{O}_{i} \in \mathcal{B}$ to project the $\mathbf{n}$ th state from the vacuum

$$
\mathcal{O}_{\mathbf{n}}^{\mathrm{opt}}=\sum_{i} v_{\mathbf{n}}^{i} \mathcal{O}_{i}
$$


The variational method may equally be applied to correlation matrices comprised of three-point functions of different interpolating fields. This would necessarily produce a better determined $\mathcal{O}_{\mathbf{n}}^{\text {opt }}$, but due to the high Wick contraction cost of using distillation we elected to perform the variational method on a correlation matrix of two-point functions, thereby fixing $\mathcal{O}_{\mathbf{n}}^{\text {opt }}$ for later use.

\section{COMPUTATIONAL DETAILS}

Our analysis considers a 350 configuration ensemble of $2+1$ flavor QCD using the clover-Wilson fermion action, where the associated gauge links are smeared by one application of the stout smearing [22] algorithm. This smearing yields a tadpole-improved tree-level clover coefficient that is nearly identical to the corresponding nonperturbative determination. The reader is referred to $[15,16]$ for a discussion regarding the gauge action used to generate this ensemble. Calculations were performed on a $32^{3} \times 64$ lattice with periodic (spatial) and antiperiodic (temporal) boundary conditions and an inverse coupling of $\beta=6.3$. In the three flavor theory, the strange quark mass was set by requiring the ratio $\left(2 M_{K^{+}}^{2}-M_{\pi^{+}}^{2}\right) / M_{\Omega^{-}}$to assume its physical value. This lattice ensemble was found to have a lattice spacing of $a=0.09840(4)$ fm via the Wilson-flow scale $w_{0}$, and with $a m_{\pi} \sim 0.176803$ yielding $a m_{\pi} L \sim 5.658$ and $m_{\pi}=356 \mathrm{MeV}$.

We explore the efficacy of four different types of operators used to interpolate ground-state nucleons from the vacuum, with particular consideration given to distillation. In this work we only study zero-momentum nucleons, polarized along the $z$ axis. A future work will study nucleons with nonzero momentum.

\section{A. Jacobi-smeared interpolator}

Prior to construction of our quark sources comprising our selected interpolators, the background gauge links are smeared via 20 applications of stout smearing with smearing parameter: $\rho_{i j}=0.08$ and $\rho_{\mu 4}=\rho_{4 \mu}=0$, where $\rho_{\mu \nu}$ quantifies the weight given to staple links aligned in the $\mu \nu$ plane when constructing the smeared links. Such gauge smearing is essential for reducing noise present in the resultant correlation functions due to source fluctuations. Before inverting the Dirac operator against the smeared sources, we apply a single iteration of stout smearing with $\rho=0.125$ to the gauge links - thereby avoiding potentially small eigenvalues in the inversion.

As a benchmark with which to compare to distillation, we begin with the simplest nucleon interpolator consistent with the nucleon $J^{P C}$

$$
\mathcal{N}(x)=\epsilon^{a b c}\left[u^{a \top}(x) C \frac{\left(1 \pm \gamma_{4}\right)}{2} \gamma_{5} d^{b}(x)\right] u^{c}(x)
$$

where $u, d$ are the two flavors of (degenerate) light quarks, $\{a, b, c\}$ color indices, $C=\gamma_{2} \gamma_{4}$ the charge conjugation matrix, and a suppressed Dirac index. The nonrelativistic projector $\left(1 \pm \gamma_{4}\right) / 2$ is included in the operator construction to reduce the noise-to-signal ratio in forward (backward) propagating states. To make contact with previous work (e.g., [15,16]) $\mathcal{N}(x)$ is smeared with 60 hits of Jacobi smearing, with width $\sigma=5.0$. We refer the reader to $[15,16]$ for an extensive analysis of the effect different Jacobi smearing parameters has on the determination of nucleon isovector charges. Herein we refer to the Jacobismeared interpolator at "Jacobi-SS", where SS indicates a smeared source and sink.

Correlation functions that employ $\mathcal{N}$ as the source/sink interpolator are constructed via application of appropriate projection operators

$$
\begin{aligned}
C^{2 \mathrm{pt}}(t) & =\sum_{\vec{x}}\left\langle\mathcal{P}_{\beta \alpha}^{2 \mathrm{pt}} \mathcal{N}_{\alpha}(\vec{x}, t) \overline{\mathcal{N}}_{\beta}(0)\right\rangle \\
C^{3 \mathrm{pt}}(t, \tau) & =\sum_{\vec{x}, \vec{z}}\left\langle\mathcal{P}_{\beta \alpha}^{3 \mathrm{pt}} \mathcal{N}_{\alpha}(\vec{x}, t) \mathcal{O}_{\Gamma}^{u-d}(\vec{z}, \tau) \overline{\mathcal{N}}_{\beta}(0)\right\rangle
\end{aligned}
$$

where $\mathcal{P}^{2 p t}=\left(1+\gamma_{4}\right) / 2$ is used to project onto the forward-propagating positive-parity nucleon, and where $\mathcal{P}^{3 p t}=\mathcal{P}^{2 p t}\left(1+i \gamma_{5} \gamma_{3}\right)$ is used for the corresponding connected insertions. The spatial sums serve to project each correlation function to zero momentum. A standard spectral decomposition demonstrates that the Dirac structure of $\mathcal{O}_{\Gamma}^{u-d}$ must be $1, \gamma_{4}, \gamma_{3} \gamma_{5}, \gamma_{1} \gamma_{2}$ to extract the scalar, vector, axial, and tensor charges, respectively. Lastly, the sequential-source method is implemented to calculate $C^{3 \mathrm{pt}}$, thereby minimizing the number of distinct inversions of the Dirac operator.

\section{B. Distilled interpolators}

We use a distillation space of rank 64, from which the perambulators and solution vectors are constructed. The distillation space on each time slice is calculated only after 10 iterations of stout smearing is applied to the gauge links with smearing factor $\rho_{i j}=0.08$ and $\rho_{\mu 4}=\rho_{4 \mu}=0$.

When expressed in a form exposing the permutational symmetry of the flavor $(\mathcal{F})$, spatial $(\mathcal{D})$ and Dirac $(\mathcal{S})$ structures, our distilled interpolators take the form

$$
\mathcal{O}=\left(\mathcal{F}_{\mathcal{P}(\mathrm{F})} \otimes \mathcal{S}_{\mathcal{P}(\mathrm{S})} \otimes \mathcal{D}_{\mathcal{P}(\mathrm{D})}\right)\left\{q_{1} q_{2} q_{3}\right\}
$$

where $\mathcal{P}(\cdots)$ expresses the symmetric $(\mathrm{S})$, mixed-symmetric (M), and antisymmetric (A) character of the given structure. Explicitly our employed distilled interpolators are
(i) $\left(N_{M} \otimes\left(\frac{1}{2}^{+}\right)_{M}^{1} \otimes D_{L=0, S}^{[0]}\right)^{J^{P}=\frac{1}{2}+}={ }^{2} S_{S} \frac{1}{2}{ }^{+}$
(ii) $\left(N_{M} \otimes\left(\frac{1}{2}^{+}\right)_{M}^{1} \otimes D_{L=0, M}^{[2]}\right)^{J^{P}=\frac{1+}{2}}={ }^{2} S_{M} \frac{1}{2}^{+}$
(iii) $\left(N_{M} \otimes\left(\frac{1}{2}^{+}\right)_{M}^{1} \otimes D_{L=0, S}^{[2]}\right)^{J^{P}=\frac{1}{2}+}={ }^{2} S_{S}^{\prime} \frac{1}{2}^{+}$
(iv) $\left(N_{M} \otimes\left(\frac{1}{2}+\right)_{M}^{1} \otimes D_{L=1, A}^{[2]}\right)^{J^{P}=\frac{1}{2}^{+}}={ }^{2} P_{A} \frac{1}{2}+$
(v) $\left(N_{M} \otimes\left(\frac{1}{2}^{+}\right)_{M}^{1} \otimes D_{L=1, M}^{[2]}\right)^{J^{P}=\frac{1}{2}+}={ }^{2} P_{M^{\frac{1}{2}}}{ }^{+}$ 
(vi) $\left(N_{M} \otimes\left(\frac{3}{2}^{+}\right)_{S}^{1} \otimes D_{L=1, M}^{[2]}\right)^{J^{P}=\frac{1+}{2}}={ }^{4} P_{M} \frac{1}{2}^{+}$

(vii) $\left(N_{M} \otimes\left(\frac{3}{2}^{+}\right)_{S}^{1} \otimes D_{L=2, M}^{[2]}\right)^{J^{P}=\frac{1+}{2}}={ }^{4} D_{M} \frac{1}{2}^{+}$

where the superscript $J^{P}$ indicates the overall spin-parity quantum numbers of the interpolator [19]. For brevity, we have expressed the interpolators in a compact spectroscopic notation ${ }^{2 S+1} L_{\mathcal{P}} J^{P}$, where $S$ represents the Dirac spin, $L$ the angular momentum induced by any derivatives, $\mathcal{P}$ the permutational symmetry of the derivatives, and $J^{P}$ the total angular momentum and parity of the interpolator. The first distilled interpolator we consider is the ${ }^{2} S_{S} \frac{1}{2}+$ interpolator, which is the closest nonrelativistic analogue of the standard nucleon interpolator given in Eq. (12).

Our first application of the variational method is to a basis of three distilled interpolators

$$
\mathcal{B}_{3}=\left\{{ }^{2} S_{S^{2}}{ }^{\frac{1}{}},{ }^{2} P_{M} \frac{1}{2}+,{ }^{4} P_{M \frac{1}{2}}+\right\}
$$

where we note ${ }^{2} P_{M}{ }^{\frac{1}{2}}+$ and ${ }^{4} P_{M \frac{1}{2}}{ }^{+}$are explicitly of hybrid character. This choice is principally motivated by [20] where it was found these hybrid interpolators, in addition to ${ }^{2} S_{S}{ }^{\frac{1}{2}}$, had predominant overlap onto the ground-state nucleon. The variational method applied to $\mathcal{B}_{3}$ leads to a variationally improved interpolator that we define to be $\hat{\mathcal{P}}_{3}$. The final interpolator we consider is found by expanding the basis $\mathcal{B}_{3}$ to include distilled interpolators that probe the radial/orbital structure of the nucleon (see above)

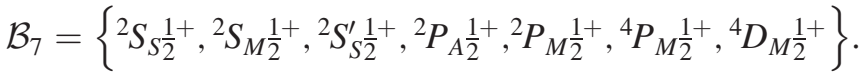

We refer to this variationally improved interpolator as $\hat{\mathcal{P}}_{7}$. The superficially redundant ${ }^{2} S_{S} \frac{1}{2}+$ and ${ }^{2} S_{S}^{\prime} \frac{1}{2}+$ interpolators, with the same spectroscopic notation but differing derivative constructions, correspond to different radial extents of the interpolator.

As outlined in Sec. II D, the construction of a variationally improved interpolator relies on careful selection of a reference time slice $t_{0}$, and the time slice $t_{Z}$ at which to evaluate the overlap factors. Dividing out the ground-state time dependence, if a single exponential were to contribute to the principal correlator, a plateau of unity should be expected. Based on the applied fits of Eq. (10), a good determination of the ground state within our basis of interpolators is thus indicated by a plateau in the rescaled correlator around unity $-A_{0} \ll 1$ and $\Delta m_{n^{\prime} 0}=m_{n^{\prime}}-m_{0} \gg 1$. For the two variationally improved interpolators we consider, we found

$$
\begin{aligned}
& \hat{\mathcal{P}}_{3} \longrightarrow\left\{t_{0}=6, t_{Z}=7\right\} \\
& \hat{\mathcal{P}}_{7} \longrightarrow\left\{t_{0}=3, t_{Z}=5\right\}
\end{aligned}
$$

led to ideal reconstruction of the original correlator.

\section{Matrix element extraction}

The extraction of matrix elements on the lattice typically proceeds by calculation of some 3-point correlator, over a range of source-sink interpolator separations, and where an external current is inserted for all intermediate times. Under the presumption of no excited-state contamination (i.e., in the limits $0 \ll \tau \ll t_{\text {sep }}$ ), the 3 -point correlator is then divided per ensemble average by a two-point correlator with the same source/sink interpolators of the same sourcesink separation. This division removes overlap factors, masses, and exponential source/sink time dependence from the matrix element signal. A plateau in this ratio, for fixed interpolator separations and varying current insertion times, should be the desired matrix element. However, as we are interested in ground-state matrix elements $\mathcal{J}_{00}$, the plateau necessarily contains contributions from matrix elements of all excited states.

\section{Correlator behavior}

Our two-point correlation function using Jacobi-smeared interpolators is defined by

$$
C_{\alpha \beta}^{2 \mathrm{pt}}(t)=\sum_{\vec{x}}\left\langle\mathcal{N}_{\alpha}(\vec{x}, t) \overline{\mathcal{N}}_{\beta}(\overrightarrow{0}, 0)\right\rangle .
$$

Inserting the nonrelativistic projector, and performing a spectral decomposition exposes the competing contributions of all states in the spectrum:

$$
C^{2 \mathrm{pt}}(t)=2 \sum_{n}\left|Z_{n}\right|^{2} e^{-M_{n} t},
$$

where the sum is only over eigenstates with quantum numbers of $\mathcal{N}$. To quantify and control excited-state contributions to $C^{2 \mathrm{pt}}$, we elect to perform a 2 -state fit of the form

$$
C_{\text {fit }}^{2 \mathrm{pt}}(t)=e^{-M_{0} t}\left[|\mathbf{a}|^{2}+|\mathbf{b}|^{2} e^{-\left(M_{1}-M_{0}\right) t}\right]
$$

for each of the four distinct interpolators we consider. The factoring of the ground-state time dependence aids in stabilizing our fits and in the determination of our extracted charges, as explained later. In this manner we obtain determinations of $M_{0}, M_{1},\left|Z_{0}\right|^{2}$ and $\left|Z_{1}\right|^{2}$, while simultaneously quantifying the efficacy of each interpolator to separate the ground state from its excitations viz. $\Delta m=$ $M_{1}-M_{0}$. The correlator behavior when using distilled interpolators is identical to that above, except for the addition of an overall volume factor $V_{3}$ due to the momentum projection at the source implied by Eq. (6).

A zero-momentum projected three-point correlation function using Jacobi-smeared interpolators is given by

$$
C_{\alpha \beta}^{3 \mathrm{pt}}\left(t_{\mathrm{sep}} ; \tau\right)=\sum_{\vec{x}} \sum_{\vec{z}}\left\langle\mathcal{N}_{\alpha}\left(\vec{x}, t_{\mathrm{sep}}\right) \mathcal{J}(\vec{z}, \tau) \overline{\mathcal{N}}_{\beta}(\overrightarrow{0}, 0)\right\rangle,
$$


where $\tau$ is the insertion time slice, restricted to the time ordering $0<\tau<t_{\text {sep }}$, and $\mathcal{J}$ the external current. An analogous spectral decomposition with the appropriate projector leads to

$$
\begin{aligned}
C^{3 \mathrm{pt}}\left(t_{\mathrm{sep}} ; \tau\right)= & \sum_{n, s} \sum_{n^{\prime}, s^{\prime}} \frac{e^{-M_{n^{\prime}}\left(t_{\mathrm{sep}}-\tau\right)} e^{-M_{n} \tau}}{4 M_{n^{\prime}} M_{n}} \\
& \times Z_{n^{\prime}} Z_{n}^{\dagger}\left\langle n^{\prime}, p^{\prime}, s^{\prime}|\mathcal{J}| n, p, s\right\rangle .
\end{aligned}
$$

Again retaining the lowest two energy eigenstates in the sum, we have

$$
\begin{aligned}
C^{3 \mathrm{pt}}\left(t_{\mathrm{sep}}, \tau\right)= & \left(\frac{\left|Z_{0}\right|^{2}}{4 M_{0}^{2}} \mathcal{J}_{00} e^{-M_{0} t_{\text {sep }}}+\frac{\left|Z_{1}\right|^{2}}{4 M_{1}^{2}} \mathcal{J}_{11} e^{-M_{1} t_{\text {sep }}}\right) \\
& +\left(\frac{Z_{0} Z_{1}^{\dagger}}{4 M_{0} M_{1}} \mathcal{J}_{01} e^{-M_{0} t_{\text {sep }}} e^{-\left(M_{1}-M_{0}\right) \tau}\right. \\
& \left.+\frac{Z_{1} Z_{0}^{\dagger}}{4 M_{0} M_{1}} \mathcal{J}_{10} e^{-M_{1} t_{\text {sep }}} e^{\left(M_{1}-M_{0}\right) \tau}\right)
\end{aligned}
$$

where $\mathcal{J}_{n^{\prime} n}=\left\langle n^{\prime}, s^{\prime}|\mathcal{J}| n, s\right\rangle$, with $n^{\prime}, n \in \mathbb{N}$. By isolating the current insertion time dependence of the three-point correlator, it becomes clear that calculation of a three-point correlation function for a single source-sink separation $t_{\text {sep }}$ is insufficient to reliably extract the matrix elements $\mathcal{J}_{00}$ and $\mathcal{J}_{11}$. As $Z_{\mathrm{n}}$ are real and $\mathcal{J}_{01}=\mathcal{J}_{10}$ for zero-momentum states, the above can be reorganized into

$$
\begin{aligned}
C^{3 \mathrm{pt}}\left(t_{\text {sep }}, \tau\right)= & \left(\frac{\left|Z_{0}\right|^{2}}{4 M_{0}^{2}} \mathcal{J}_{00} e^{-M_{0} t_{\text {sep }}}+\frac{\left|Z_{1}\right|^{2}}{4 M_{1}^{2}} \mathcal{J}_{11} e^{-M_{1} t_{\text {sep }}}\right) \\
& +\frac{Z_{0} Z_{1}}{2 M_{0} M_{1}} \mathcal{J}_{01} e^{-\frac{\left(M_{1}+M_{0}\right)}{2} t_{\text {sep }}} \\
& \times \cosh \left[\left(M_{1}-M_{0}\right)\left(\tau-\frac{t_{\text {sep }}}{2}\right)\right] .
\end{aligned}
$$

We then apply a 2-state fit to the three-point correlation functions,

$$
\begin{aligned}
C_{\mathrm{fit}}^{3 \mathrm{pt}}\left(t_{\mathrm{sep}}, \tau\right)= & e^{-M_{0} t_{\mathrm{sep}}}\left(\mathcal{A}+\mathcal{B} e^{-\Delta m t_{\mathrm{sep}}}\right. \\
& \left.+\mathcal{C} e^{-\Delta m_{\text {sep }}^{2}} \cosh \left[\Delta m\left(\tau-\frac{t_{\mathrm{sep}}}{2}\right)\right]\right)
\end{aligned}
$$

where $\tau$ is the current insertion time and $\Delta m=M_{1}-M_{0}$. We note that we retain $M_{0}$ and $M_{1}$ as parameters in our fit, rather than the difference $\Delta m$. As with the functional form employed to fit the two-point correlation functions, we factor the ground-state time dependence from the functional form of the three-point fits. This factoring makes manifest the desired ground-state matrix element in the limits $0 \ll$ $\tau \ll t_{\text {sep }}$. The correlator behavior when using distilled interpolators is again identical to that above, except for the addition of an overall volume factor $V_{3}$ to Eq. (20).

Determining the precise relationship between the fitted parameters to extract the ground-state matrix element $\mathcal{J}_{00}$, requires a more detailed look at our interpolators. Although the constructed distilled interpolators contain no free spinor indices, our use of positive-parity nucleons polarized along the $z$ direction can be viewed as the standard application of projectors on the nucleon interpolating field of Eq. (12). At zero momentum we again have

$$
\begin{aligned}
C^{2 \mathrm{pt}}(t) & =\sum_{\vec{x}}\left\langle\mathcal{P}_{\beta \alpha}^{2 \mathrm{pt}} \mathcal{N}_{\alpha}(\vec{x}, t) \overline{\mathcal{N}}_{\beta}(0)\right\rangle \\
C^{3 \mathrm{pt}}\left(t_{\mathrm{sep}}, \tau\right) & =\sum_{\vec{x}, \vec{z}}\left\langle\mathcal{P}_{\beta \alpha}^{3 \mathrm{pt}} \mathcal{N}_{\alpha}\left(\vec{x}, t_{\mathrm{sep}}\right) \mathcal{O}_{\Gamma}^{u-d}(\vec{z}, \tau) \overline{\mathcal{N}}_{\beta}(0)\right\rangle .
\end{aligned}
$$

Proceeding with the spectral decomposition of $C^{2 \mathrm{pt}}$ we have

$$
\begin{aligned}
C^{2 \mathrm{pt}}\left(t_{\mathrm{sep}}\right)= & \sum_{n, s} \frac{e^{-M_{n} t_{\mathrm{sep}}}}{2 M_{n}} \mathcal{P}_{\beta \alpha}^{2 \mathrm{pt}} \\
& \times\left\langle\Omega\left|\mathcal{N}_{\alpha}\right| n, p, s\right\rangle\left\langle n, p, s\left|\mathcal{N}_{\beta}^{\dagger}\right| \Omega\right\rangle \\
= & \sum_{n, s} \frac{\left|Z_{n}\right|^{2} e^{-M_{n} t_{\mathrm{sep}}}}{2 M_{n}} \mathcal{P}_{\beta \alpha}^{2 \mathrm{pt}} u_{\alpha}^{n}(\overrightarrow{0}, s) \bar{u}_{\beta}^{n}(\overrightarrow{0}, s) \\
= & \sum_{n} \frac{\left|Z_{n}\right|^{2} e^{-M_{n} t_{\mathrm{sep}}}}{2 M_{n}} \operatorname{Tr}\left[\mathcal{P}^{2 \mathrm{pt}}\left(-i \not \not_{E}+M_{n}\right)\right],
\end{aligned}
$$

from which it is easily shown that the constant coefficients of the 2-state fit applied to our two-point correlators are of the form $|\mathbf{a}|^{2}=2\left|Z_{0}\right|^{2}$ and $|\mathbf{b}|^{2}=2\left|Z_{1}\right|^{2}$.

The spectral decomposition of $C^{3 p t}$ proceeds analogously,

$$
\begin{aligned}
C^{3 \mathrm{pt}}\left(t_{\mathrm{sep}}, \tau\right) & =\sum_{n, s} \sum_{n^{\prime}, s^{\prime}} \frac{e^{-M_{n^{\prime}}\left(t_{\mathrm{sep}}-\tau\right)} e^{-M_{n} \tau}}{4 M_{n^{\prime}} M_{n}} Z_{n^{\prime}} Z_{n}^{\dagger} \mathcal{P}_{\beta \alpha}^{3 \mathrm{pt}} u_{\alpha}^{n^{\prime}}\left(\overrightarrow{0}, s^{\prime}\right) \bar{u}_{\beta}^{n}(\overrightarrow{0}, s)\left\langle n^{\prime}, p^{\prime}, s^{\prime}|\mathcal{J}| n, p, s\right\rangle \\
& =\sum_{n, s} \sum_{n^{\prime}, s^{\prime}} \frac{e^{-M_{n^{\prime}}\left(t_{\mathrm{sep}}-\tau\right)} e^{-M_{n} \tau}}{4 M_{n^{\prime}} M_{n}} Z_{n^{\prime}} Z_{n}^{\dagger} \mathcal{P}_{\beta \alpha}^{3 \mathrm{pt}} u_{\alpha}^{n^{\prime}}\left(\overrightarrow{0}, s^{\prime}\right) \bar{u}_{\beta}^{n}(\overrightarrow{0}, s) \bar{u}_{\rho}^{n^{\prime}}\left(\overrightarrow{0}, s^{\prime}\right) \mathcal{J}_{\rho \sigma} u_{\sigma}^{n}(\overrightarrow{0}, s) \\
& =\sum_{n^{\prime}, n} \frac{Z_{n^{\prime}} Z_{n}^{\dagger}}{4 M_{n^{\prime}} M_{n}} e^{-M_{n^{\prime}}\left(t_{\mathrm{sep}}-\tau\right)} e^{-M_{n} \tau} \operatorname{Tr}\left[\mathcal{P}^{3 \mathrm{pt}}\left(-i \not \not_{E}{ }^{\prime}+M_{n^{\prime}}\right) \mathcal{J}_{n^{\prime} n}\left(-i \not \not_{E}+M_{n}\right)\right] .
\end{aligned}
$$


It then follows $\mathcal{A}=2 g_{\Gamma}^{00}\left|Z_{0}\right|^{2}$ and $\mathcal{B}=2 g_{\Gamma}^{11}\left|Z_{1}\right|^{2}$. We can then extract the ground-state matrix element via

$$
g_{00}^{\Gamma}=\mathcal{A} /|\mathbf{a}|^{2} .
$$

To extract the masses, overlap factors and matrix elements from our data, we perform simultaneous fits to the twopoint and three-point correlation functions according to Eqs. (19) and (22), respectively.

\section{RESULTS}

We compute the two-point functions averaged over three source positions. The extraction for the matrix elements is obtained from three-point functions for $t_{\text {sep }} \in\{8,12,16\}$, and $\tau \in\left[0, t_{\text {sep }}-1\right]$. Rather than view the calculated twopoint correlation functions directly, we judge the quality of the two-point correlators for each interpolator by plotting the effective mass

$$
M_{\mathrm{eff}}(t+0.5)=\frac{1}{a} \ln \frac{C^{2 \mathrm{pt}}(t)}{C^{2 \mathrm{pt}}(t+1)}
$$

as a function of the source-sink separation $t$. Calculating each two-point correlator, averaged over three different source positions, leads to the effective masses seen in Fig. 1(b). The lack of a plateau in the effective mass of the Jacobi-SS interpolator until $t \sim 10$ is indicative of excitedstate contamination for source-sink separations of $\lesssim 1 \mathrm{fm}$. Use of the ${ }^{2} S_{S_{2}}{ }^{+}$distilled interpolator leads to an earlier onset of a plateau in the effective mass, with statistical uncertainty that is at least $50 \%$ smaller than that of the Jacobi-SS interpolator. This plateau is seen to begin for

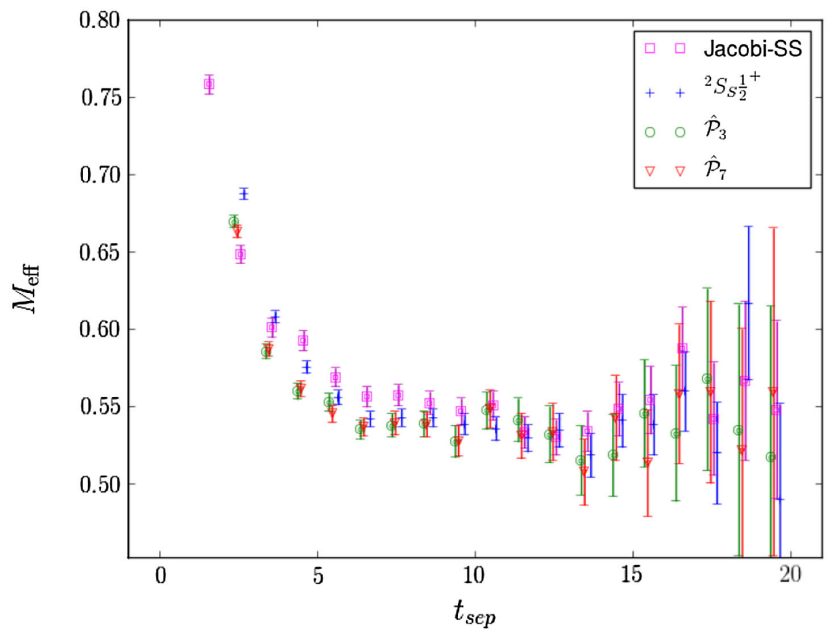

(a) $t \sim 6$, or $\sim 0.6 \mathrm{fm}$. It is also worth noting that the expected exponentially increasing noise in the nucleon effective mass is substantially suppressed at larger source-sink separations, when compared to the Jacobi-SS interpolator.

Use of a variationally improved interpolator derived from bases of distilled interpolators, $\left(\hat{\mathcal{P}}_{3}\right.$ or $\left.\hat{\mathcal{P}}_{7}\right)$, leads to a more rapid exponential decay of excited states at early Euclidean times. The effective mass induced by the $\hat{\mathcal{P}}_{3}$ interpolator exhibits a plateau that has nearly the same statistical precision as that of the ${ }^{2} S_{S^{2}}{ }^{1+}$ interpolator, while the excited states are seen to decay more rapidly for $t<5$. Expanding the basis of distilled interpolators, the $\hat{\mathcal{P}}_{7}$ interpolator leads to an even more rapid decay of excited states for $t<5$, consistent with a variationally improved interpolator receiving excited-state contributions from states higher in energy than those within the basis. As with the ${ }^{2} S_{S_{2}}{ }^{+}$and $\hat{\mathcal{P}}_{3}$ interpolators, the effective mass of $\hat{\mathcal{P}}_{7}$ begins around $t=6$, however the plateau is noticeably lower than the former. In general, the statistical precision of all distilled interpolators appears to be comparable, except for large Euclidean times wherein the determination of the variationally improved interpolators becomes increasingly uncertain. We attribute this increased uncertainty to stem from the variationally improved interpolators being relatively unconstrained at large source-sink separations, where elements of the correlation matrix [Eq. (8)] are themselves dominated by noise.

\section{A. Two-state analysis}

To quantify the discussion above and to guide our future simultaneous fits to the two- and three-point functions, we explore the efficacy of one- and two-state fits have in

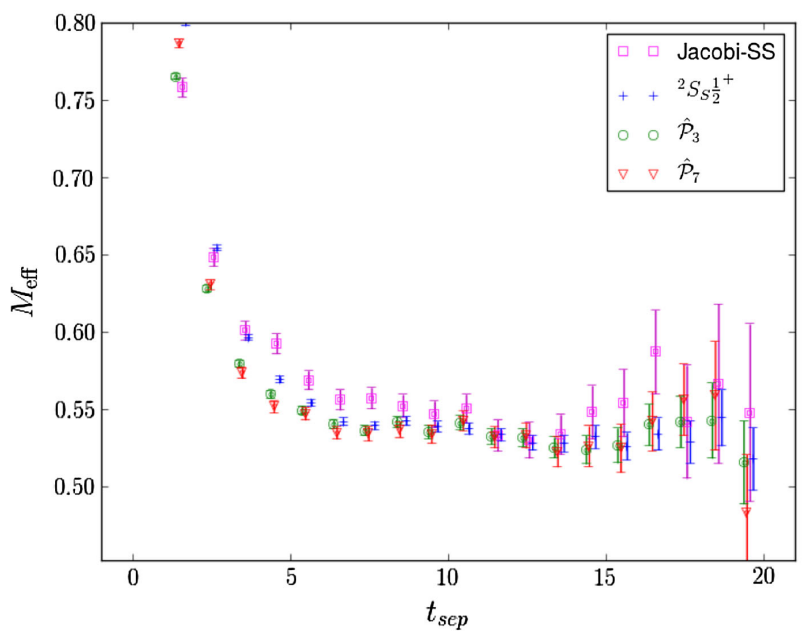

(b)

FIG. 1. Nucleon effective mass when using Jacobi-SS (purple) and distilled interpolators constructed from 32 (a) and 64 (b) eigenvectors. The distilled interpolators ${ }^{2} S_{S^{2}}{ }^{+}{ }^{+}$(blue), $\hat{\mathcal{P}}_{3}$ (green), and $\hat{\mathcal{P}}_{7}$ (red) demonstrate a plateau in the effective mass as early as $t_{\text {sep }}=6$, but the statistical uncertainty is comparable to that of the Jacobi-SS when 32 eigenvectors are used. Considerable improvement in statistics is seen for the case of 64 eigenvectors. 
TABLE I. Parameters of a single-state fit to the two-point correlators, where the distilled interpolators were constructed from 64 eigenvectors. As the initial time slice over which fits are performed is made larger, the determined ground-state mass, as expected, is found to decrease. The ground-state mass of the Jacobi-SS interpolator is seen to converge toward $M_{0} \sim 0.55$, while for the distilled interpolators the ground-state mass appears to converge towards $M_{0} \sim 0.535$. Errors are purely statistical.

\begin{tabular}{lcccc}
\hline \hline$\hat{\mathcal{O}}$ & $t_{\text {fit }}$ & $|\mathbf{a}|^{2}$ & $M_{0}$ & $\chi_{r}^{2}$ \\
\hline Jacobi-SS & {$[4,16]$} & $5.032(095) \times 10^{-08}$ & $0.563(4)$ & 4.334 \\
& {$[5,16]$} & $4.701(100) \times 10^{-08}$ & $0.556(3)$ & 1.458 \\
& {$[6,16]$} & $4.516(122) \times 10^{-08}$ & $0.551(4)$ & 0.932 \\
& {$[7,16]$} & $4.450(148) \times 10^{-08}$ & $0.550(4)$ & 0.977 \\
${ }^{2} S_{S^{2}}{ }^{+}$ & {$[4,16]$} & $1.627(011) \times 10^{-02}$ & $0.548(1)$ & 20.77 \\
& {$[5,16]$} & $1.552(011) \times 10^{-02}$ & $0.542(1)$ & 6.314 \\
& {$[6,16]$} & $1.500(013) \times 10^{-02}$ & $0.538(1)$ & 2.354 \\
& {$[7,16]$} & $1.483(016) \times 10^{-02}$ & $0.537(1)$ & 2.237 \\
$\hat{\mathcal{P}}_{3}$ & {$[4,16]$} & $1.147(07) \times 10^{+00}$ & $0.544(1)$ & 9.448 \\
& {$[5,16]$} & $1.108(08) \times 10^{+00}$ & $0.540(1)$ & 3.461 \\
& {$[6,16]$} & $1.080(10) \times 10^{+00}$ & $0.537(1)$ & 1.705 \\
& {$[7,16]$} & $1.069(13) \times 10^{+00}$ & $0.536(2)$ & 1.620 \\
$\hat{\mathcal{P}}_{7}$ & {$[4,16]$} & $1.045(09) \times 10^{+00}$ & $0.540(1)$ & 3.094 \\
& {$[5,16]$} & $1.021(10) \times 10^{+00}$ & $0.537(2)$ & 1.509 \\
& {$[6,16]$} & $0.999(12) \times 10^{+00}$ & $0.535(2)$ & 0.613 \\
& {$[7,16]$} & $0.999(15) \times 10^{+00}$ & $0.535(2)$ & 0.688 \\
\hline \hline
\end{tabular}

describing the calculated effective masses. All fits are restricted such that $2 \leq t_{\text {fit }} \leq 16$ thereby avoiding contact terms in the clover Wilson action and a collective fluctuation of the effective masses for $t \gtrsim 16$. The results of these fits are collected in Tables I and II.
Although single-state fits make explicit the improvements gained by using distillation over standard Jacobi smearing, notably a $\sim 3 \%$ difference in the determination of the ground-state mass, the improvements at this stage hardly seem worth the cost of constructing the required distillation basis. By including a second state in the fits performed to the two-point correlators, the gains produced by distillation are quite encouraging.

The inclusion of a second state in fits to the two-point correlators yields slightly smaller determinations for the ground-state mass and overlap factors when using the Jacobi, ${ }^{2} S_{S}{ }^{1}{ }^{+}$, and $\hat{\mathcal{P}}_{3}$ interpolators, while little change is seen in the $\hat{\mathcal{P}}_{7}$ interpolator. The most striking difference between the interpolators comes in the determination of the first-excited state mass. The determined mass gaps are

$$
\Delta m=\left(M_{1}-M_{0}\right) \simeq \begin{cases}0.497(80) & \text { Jacobi-SS } \\ 0.714(30) & { }^{2} S_{S^{\frac{1}{2}}} \\ 0.730(51) & \hat{\mathcal{P}}_{3} \\ 0.895(80) & \hat{\mathcal{P}}_{7}\end{cases}
$$

Evidently distillation and the variational method lead to greater elimination of excited-state contributions to the two-point correlators, where the mass gap is $\sim 44 \%, \sim 47 \%$, and $\sim 80 \%$ larger for the ${ }^{2} S_{S_{2}}{ }^{+}, \hat{\mathcal{P}}_{3}$, and $\hat{\mathcal{P}}_{7}$ interpolators, respectively, when compared to the Jacobi-SS interpolator. The compounded improvements of the variational method applied to our bases of distilled interpolators is entirely consistent with [23].

TABLE II. Parameters of two-state fits to the two-point correlators, where the distilled interpolators were constructed from 64 eigenvectors. There is general agreement in determination of the ground-state mass, while the mass gap $\Delta m=M_{1}-M_{0}$ is seen to become ever larger as distillation, and the variational analysis applied to a basis of distilled interpolators, is used in place of the Jacobi-SS interpolator. Fits performed over larger source-sink separations were increasingly consistent with single-state fits, and are not considered further. Errors are purely statistical.

\begin{tabular}{lcccccc}
\hline \hline$\hat{\mathcal{O}}$ & $t_{\text {fit }}$ & \multicolumn{1}{c}{$|\mathbf{a}|^{2}$} & $M_{0}$ & $|\mathbf{b}|^{2}$ & $M_{1}$ & $\chi_{r}^{2}$ \\
\hline Jacobi-SS & {$[2,16]$} & $4.12(25) \times 10^{-08}$ & $0.543(6)$ & $3.70(25) \times 10^{-08}$ & $1.04(08)$ & 0.842 \\
& {$[3,16]$} & $3.81(42) \times 10^{-08}$ & $0.536(9)$ & $3.12(35) \times 10^{-08}$ & $0.91(11)$ & 0.753 \\
& {$[4,16]$} & $4.14(48) \times 10^{-08}$ & $0.54(01)$ & $5.3(5.9) \times 10^{-08}$ & $1.13(42)$ & 0.667 \\
${ }^{2} S_{S^{2}}{ }^{+}$ & {$[2,16]$} & $1.45(02) \times 10^{-02}$ & $0.536(2)$ & $1.69(06) \times 10^{-02}$ & $1.25(03)$ & 1.535 \\
& {$[3,16]$} & $1.43(03) \times 10^{-02}$ & $0.534(2)$ & $1.35(14) \times 10^{-02}$ & $1.14(06)$ & 1.268 \\
& {$[4,16]$} & $1.42(04) \times 10^{-02}$ & $0.534(3)$ & $1.31(52) \times 10^{-02}$ & $1.13(15)$ & 1.407 \\
$\hat{\mathcal{P}}_{3}$ & {$[2,16]$} & $1.06(2) \times 10^{+00}$ & $0.535(2)$ & $9.26(54) \times 10^{-01}$ & $1.265(51)$ & 1.310 \\
& {$[3,16]$} & $1.04(19) \times 10^{+00}$ & $0.533(16)$ & $0.70(3.19) \times 10^{+00}$ & $1.12(1.51)$ & 1.076 \\
& {$[4,16]$} & $1.03(4) \times 10^{+00}$ & $0.533(4)$ & $5.77(3.17) \times 10^{-01}$ & $1.05(23)$ & 1.127 \\
$\hat{\mathcal{P}}_{7}$ & {$[2,16]$} & $1.00(1) \times 10^{+00}$ & $0.535(2)$ & $1.08(13) \times 10^{+00}$ & $1.43(08)$ & 0.737 \\
& {$[3,16]$} & $0.98(2) \times 10^{+00}$ & $0.533(2)$ & $0.68(24) \times 10^{+00}$ & $1.23(17)$ & 0.668 \\
& {$[4,16]$} & $0.98(4) \times 10^{+00}$ & $0.533(3)$ & $0.48(53) \times 10^{+00}$ & $1.13(39)$ & 0.729 \\
\hline \hline
\end{tabular}


TABLE III. Results of simultaneous fits to the two-point and three-point correlators with scalar current insertion. The functional forms are given in Eqs. (19) and (22). The distilled interpolators were constructed from 64 eigenvectors.

\begin{tabular}{|c|c|c|c|c|c|c|c|c|c|}
\hline & $\tau_{\text {buff }}$ & $\mathcal{A}$ & $\mathcal{B}$ & $\mathcal{C}$ & $M_{0}$ & $M_{1}$ & ||$^{2}$ & $|\mathbf{b}|^{2}$ & $g_{S, \text { bare }}^{u-d}$ \\
\hline $\mathrm{co}$ & $\begin{array}{l}1 \\
2 \\
3 \\
4\end{array}$ & $\begin{array}{l}3.72(40) \times 10^{-08} \\
3.75(44) \times 10^{-08} \\
3.86(51) \times 10^{-08} \\
3.28(65) \times 10^{-08}\end{array}$ & $\begin{array}{l}2.57(3.12) \times 10^{-07} \\
1.33(2.46) \times 10^{-07} \\
1.54(2.87) \times 10^{-07} \\
2.08(3.09) \times 10^{-07}\end{array}$ & $\begin{array}{l}-2.93(28) \times 10^{-08} \\
-2.63(47) \times 10^{-08} \\
-3.08(95) \times 10^{-08} \\
0.34(3.47) \times 10^{-08}\end{array}$ & $\begin{array}{l}0.546(5) \\
0.544(5) \\
0.543(6) \\
0.543(6)\end{array}$ & $\begin{array}{l}1.087(77) \\
1.061(76) \\
1.054(78) \\
1.044(75)\end{array}$ & $\begin{array}{l}4.26(20) \times 10^{-08} \\
4.17(22) \times 10^{-08} \\
4.13(23) \times 10^{-08} \\
4.11(23) \times 10^{-08}\end{array}$ & $\begin{array}{l}3.72(26) \times 10^{-08} \\
3.71(25) \times 10^{-08} \\
3.75(25) \times 10^{-08} \\
3.75(24) \times 10^{-08}\end{array}$ & $\begin{array}{l}0.87(10) \\
0.90(11) \\
0.94(13) \\
0.80(16)\end{array}$ \\
\hline$S_{S} \frac{1}{2}+$ & $\begin{array}{l}1 \\
2 \\
3 \\
4\end{array}$ & $\begin{array}{l}1.54(04) \times 10^{-02} \\
1.54(05) \times 10^{-02} \\
1.52(05) \times 10^{-02} \\
1.52(06) \times 10^{-02}\end{array}$ & $\begin{array}{l}0.48(1.08) \times 10^{-01} \\
0.47(1.23) \times 10^{-01} \\
1.27(1.38) \times 10^{-01} \\
0.18(1.41) \times 10^{-01}\end{array}$ & $\begin{array}{r}-9.97(27) \times 10^{-03} \\
-1.00(06) \times 10^{-02} \\
-1.05(13) \times 10^{-02} \\
-4.3(5.5) \times 10^{-03}\end{array}$ & $\begin{array}{l}0.537(1) \\
0.537(1) \\
0.537(1) \\
0.536(1)\end{array}$ & $\begin{array}{l}1.2 \\
1.2 \\
1.25 \\
1.2\end{array}$ & $\begin{array}{l}1.46(02) \times 10^{-02} \\
1.46(02) \times 10^{-02} \\
1.46(02) \times 10^{-02} \\
1.46(02) \times 10^{-02}\end{array}$ & $\begin{array}{l}\partial^{-02} \\
\partial^{-02} \\
\partial^{-02} \\
\partial^{-02}\end{array}$ & 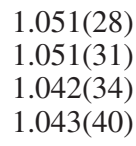 \\
\hline$\hat{P}_{3}$ & $\begin{array}{l}1 \\
2 \\
3 \\
4\end{array}$ & $\begin{array}{l}1.10(3) \times 10^{+00} \\
1.08(4) \times 10^{+00} \\
1.07(4) \times 10^{+00} \\
1.08(5) \times 10^{+00}\end{array}$ & $\begin{array}{r}5.69(12.45) \times 10^{+00} \\
8.88(12.76) \times 10^{+00} \\
18.0(16.1) \times 10^{+00} \\
4.41(15.26) \times 10^{+00}\end{array}$ & $\begin{array}{r}-6.88(22) \times 10^{-01} \\
-6.36(56) \times 10^{-01} \\
-7.08(1.20) \times 10^{-01} \\
-0.86(4.93) \times 10^{-01}\end{array}$ & $\begin{array}{l}0.537(1) \\
0.537(1) \\
0.537(1) \\
0.536(2)\end{array}$ & $\begin{array}{l}1.282(41) \\
1.268(45) \\
1.277(46) \\
1.270(46)\end{array}$ & $\begin{array}{l}1.07(1) \times 10^{+00} \\
1.07(1) \times 10^{+00} \\
1.07(1) \times 10^{+00} \\
1.06(1) \times 10^{+00}\end{array}$ & $\begin{array}{l}9.17(48)> \\
9.29(51)> \\
9.25(50)\end{array}$ & $\begin{array}{l}1.016(37) \\
1.006(40) \\
1.013(47)\end{array}$ \\
\hline$\hat{\mathcal{P}}_{7}$ & $\begin{array}{l}1 \\
2 \\
3 \\
4\end{array}$ & $\begin{array}{l}1.08(5) \times 10^{+00} \\
1.06(4) \times 10^{+00} \\
1.05(5) \times 10^{+00} \\
1.05(6) \times 10^{+00}\end{array}$ & $\begin{array}{l}30.5(65.3) \times 10^{+00} \\
42.4(48.4) \times 10^{+00} \\
94.8(73.0) \times 10^{+00} \\
57.0(78.0) \times 10^{+00}\end{array}$ & $\begin{array}{r}-6.09(29) \times 10^{-01} \\
-5.43(80) \times 10^{-01} \\
-0.73(20) \times 10^{+00} \\
0.6(1.1) \times 10^{+00}\end{array}$ & $\begin{array}{l}0.536(2) \\
0.536(2) \\
0.536(2) \\
0.536(2)\end{array}$ & $\begin{array}{l}1.440(69) \\
1.416(71) \\
1.442(72) \\
1.443(77)\end{array}$ & $\begin{array}{l}1.00(1) \times 10^{+00} \\
1.00(1) \times 10^{+00} \\
1.00(1) \times 10^{+00} \\
1.00(1) \times 10^{+00}\end{array}$ & $\begin{array}{l}1.09(11) \times 10^{+00} \\
1.06(11) \times 10^{+00} \\
1.10(12) \times 10^{+00} \\
1.10(12) \times 10^{+00}\end{array}$ & $\begin{array}{l}1.061(43) \\
1.051(46) \\
1.045(55)\end{array}$ \\
\hline
\end{tabular}

TABLE IV. Results of simultaneous fits to the two-point and three-point correlators with axial current insertion. The distilled interpolators were constructed from 64 eigenvectors. The functional forms are given in Eqs. (19) and (22).

\begin{tabular}{|c|c|c|c|c|c|c|c|c|c|}
\hline$\hat{\mathcal{O}}$ & $\tau_{\text {buff }}$ & $\mathcal{A}$ & $\mathcal{B}$ & $\mathcal{C}$ & $M_{0}$ & $M_{1}$ & $|\mathbf{a}|^{2}$ & $|\mathbf{b}|^{2}$ & $g_{A, \text { bare }}^{u-d}$ \\
\hline Jacobi-SS & $\begin{array}{l}1 \\
2 \\
3 \\
4\end{array}$ & $\begin{array}{l}4.92(32) \times 10^{-08} \\
5.00(33) \times 10^{-08} \\
5.03(33) \times 10^{-08} \\
5.37(36) \times 10^{-08}\end{array}$ & $\begin{array}{l}.65(6.23) \times 10^{-08} \\
3.23(7.15) \times 10^{-08} \\
6.83(6.78) \times 10^{-08} \\
1.54(1.15) \times 10^{-07}\end{array}$ & $\begin{array}{l}-6.66(1.32) \times 10^{-09} \\
-7.66(2.12) \times 10^{-09} \\
-1.30(0.44) \times 10^{-08} \\
-4.00(1.69) \times 10^{-08}\end{array}$ & $\begin{array}{l}0.539(5) \\
0.540(6) \\
0.540(6) \\
0.542(6)\end{array}$ & $\begin{array}{l}0.995(62) \\
1.010(67) \\
0.999(66) \\
1.032(75)\end{array}$ & $\begin{array}{l}3.93(23) \times 10^{-08} \\
3.96(23) \times 10^{-08} \\
3.95(23) \times 10^{-08} \\
4.08(24) \times 10^{-08}\end{array}$ & $\begin{array}{l}3.76(20) \times 10^{-08} \\
3.81(21) \times 10^{-08} \\
3.74(21) \times 10^{-08} \\
3.70(23) \times 10^{-08}\end{array}$ & $\begin{array}{l}1.253(52) \\
1.261(51) \\
1.272(54) \\
1.315(59)\end{array}$ \\
\hline${ }^{2} S_{S} \frac{1}{2}^{+}$ & $\begin{array}{l}1 \\
2 \\
3 \\
4\end{array}$ & $\begin{array}{l}1.95(03) \times 10^{-02} \\
1.96(03) \times 10^{-02} \\
1.96(03) \times 10^{-02} \\
1.98(04) \times 10^{-02}\end{array}$ & $\begin{array}{l}-2.52(5.57) \times 10^{-02} \\
-3.03(5.78) \times 10^{-02} \\
-1.77(5.85) \times 10^{-02} \\
-0.55(6.32) \times 10^{-02}\end{array}$ & $\begin{array}{l}-1.38(0.11) \times 10^{-03} \\
-1.33(0.23) \times 10^{-03} \\
-1.70(0.54) \times 10^{-03} \\
-6.11(2.61) \times 10^{-03}\end{array}$ & $\begin{array}{l}0.536(1) \\
0.536(1) \\
0.536(1) \\
0.536(1)\end{array}$ & $\begin{array}{l}1.242(28) \\
1.244(28) \\
1.247(28) \\
1.253(28)\end{array}$ & $\begin{array}{l}1.46(02) \times 10^{-02} \\
1.46(02) \times 10^{-02} \\
1.46(02) \times 10^{-02} \\
1.46(02) \times 10^{-02}\end{array}$ & $\begin{array}{l}1.67(05) \times 10^{-02} \\
1.68(05) \times 10^{-02} \\
1.68(06) \times 10^{-02} \\
1.69(06) \times 10^{-02}\end{array}$ & $\begin{array}{l}1.342(16) \\
1.343(16) \\
1.344(16) \\
1.357(17)\end{array}$ \\
\hline$\hat{\mathcal{P}}_{3}$ & $\begin{array}{l}1 \\
2 \\
3 \\
4\end{array}$ & $\begin{array}{l}1.41(2) \times 10^{+00} \\
1.41(3) \times 10^{+00} \\
1.42(3) \times 10^{+00} \\
1.43(3) \times 10^{+00}\end{array}$ & $\begin{array}{l}-1.06(4.54) \times 10^{+00} \\
-1.86(5.26) \times 10^{+00} \\
-1.14(5.41) \times 10^{+00} \\
-0.21(5.87) \times 10^{+00}\end{array}$ & $\begin{array}{l}-2.11(0.10) \times 10^{-01} \\
-2.28(0.22) \times 10^{-01} \\
-2.67(0.51) \times 10^{-01} \\
-5.11(2.37) \times 10^{-01}\end{array}$ & $\begin{array}{l}0.535(1) \\
0.535(1) \\
0.535(1) \\
0.536(1)\end{array}$ & $\begin{array}{l}1.250(40) \\
1.263(43) \\
1.267(44) \\
1.276(46)\end{array}$ & $\begin{array}{l}1.06(1) \times 10^{+00} \\
1.06(1) \times 10^{+00} \\
1.06(1) \times 10^{+00} \\
1.06(1) \times 10^{+00}\end{array}$ & $\begin{array}{l}9.08(43) \times 10^{-01} \\
9.22(48) \times 10^{-01} \\
9.24(49) \times 10^{-01} \\
9.32(51) \times 10^{-01}\end{array}$ & $\begin{array}{l}1.329(16) \\
1.332(17) \\
1.334(17) \\
1.340(19)\end{array}$ \\
\hline$\hat{\mathcal{P}}_{7}$ & $\begin{array}{l}1 \\
2 \\
3 \\
4\end{array}$ & $\begin{array}{l}1.33(3) \times 10^{+00} \\
1.35(3) \times 10^{+00} \\
1.35(3) \times 10^{+00} \\
1.35(3) \times 10^{+00}\end{array}$ & $\begin{array}{l}12.5(18.2) \times 10^{+00} \\
11.5(26.9) \times 10^{+00} \\
16.4(27.8) \times 10^{+00} \\
23.7(29.5) \times 10^{+00}\end{array}$ & $\begin{array}{l}-2.51(0.13) \times 10^{-01} \\
-2.87(0.38) \times 10^{-01} \\
-2.94(0.91) \times 10^{-01} \\
-6.39(5.15) \times 10^{-01}\end{array}$ & $\begin{array}{l}0.535(1) \\
0.535(2) \\
0.535(2) \\
0.535(2)\end{array}$ & $\begin{array}{l}1.403(58) \\
1.442(72) \\
1.451(76) \\
1.443(77)\end{array}$ & $\begin{array}{l}9.96(11) \times 10^{-01} \\
9.99(12) \times 10^{-01} \\
1.00(1) \times 10^{+00} \\
1.00(1) \times 10^{+00}\end{array}$ & $\begin{array}{l}1.04(9) \times 10^{+00} \\
1.10(12) \times 10^{+00} \\
1.11(13) \times 10^{+00} \\
1.10(12) \times 10^{+00}\end{array}$ & $\begin{array}{l}1.339(20) \\
1.347(22) \\
1.345(21) \\
1.346(22)\end{array}$ \\
\hline
\end{tabular}

\section{B. Results for $g_{S}^{u-d}, g_{A}^{u-d}, g_{T}^{u-d}, g_{V}^{u-d}$}

In this section we seek a more reliable determination of the masses, overlap factors, and likewise nucleon matrix elements, by performing simultaneous fits to three-point and two-point correlators with interpolator $\mathcal{O}$ and current structure $\Gamma$. We fix the window over which the two-point correlators are fit to be $t_{2 \mathrm{pt}}^{\mathrm{fit}} \in[2,16]$, while several threepoint fit windows are studied. The three-point fit windows are identified by $\tau_{\text {buff }}$-that is for a given $t_{\text {sep }}, \tau_{\text {fit }} \in\left[0+\tau_{\text {buff }}\right.$, $\left.t_{\text {sep }}-\tau_{\text {buff }}\right]$.

To illustrate the extracted isovector charges and to quantify the degree of excited-state contamination, we plot an effective charge defined as

$$
g_{\Gamma, \mathrm{eff}}\left(t_{\mathrm{sep}}, \tau\right)=\frac{C_{\Gamma}^{3 \mathrm{pt}}\left(t_{\mathrm{sep}}, \tau\right)}{C_{\mathrm{fit}}^{2 \mathrm{pt}}\left(t_{\mathrm{sep}}\right)}
$$

where $C_{\Gamma}^{3 \mathrm{pt}}\left(t_{\mathrm{sep}}, \tau\right)$ is the three-point correlation function for a given source-sink separation and current insertion time, and $C_{\mathrm{fit}}^{2 \mathrm{pt}}\left(t_{\mathrm{sep}}\right)$ is the corresponding best fit applied to the two-point function of the same interpolators and source-sink separation. Errors on the effective charges are computed via a simultaneous jackknife resampling of the two-point fit and three-point correlator, to account for the correlations between fitted parameters. Shown together with the effective charges are our extracted values for the isovector charges using the methodology described above. 
Error (gray) bands of the extracted charges (black line) are determined through the ratio of fit parameters $\mathcal{A} /|\mathbf{a}|^{2}$, likewise accounting for parameter covariance. We highlight that nearly the same masses for the ground- and first-excited states are found from the 2-state fits to the two-point correlators and from the simultaneous fits to the two- and three-point correlators.

\section{1. $g_{S}^{u-d}$}

Coupling of the isovector scalar current $S^{3}=\bar{q} \frac{\tau^{3}}{2} q$ to the Jacobi-SS interpolator $\mathcal{N}_{\alpha}$ produces an effective matrix element (denoted here as $\mathcal{M}_{S}^{\text {eff }}$ ) that is determined to within no less than $\sim 10 \%$ uncertainty. Although $\mathcal{M}_{S}^{\text {eff }}$ is symmetric about the midpoint $\tau-t_{\text {sep }} / 2$ for $t_{\text {sep }}=8$,

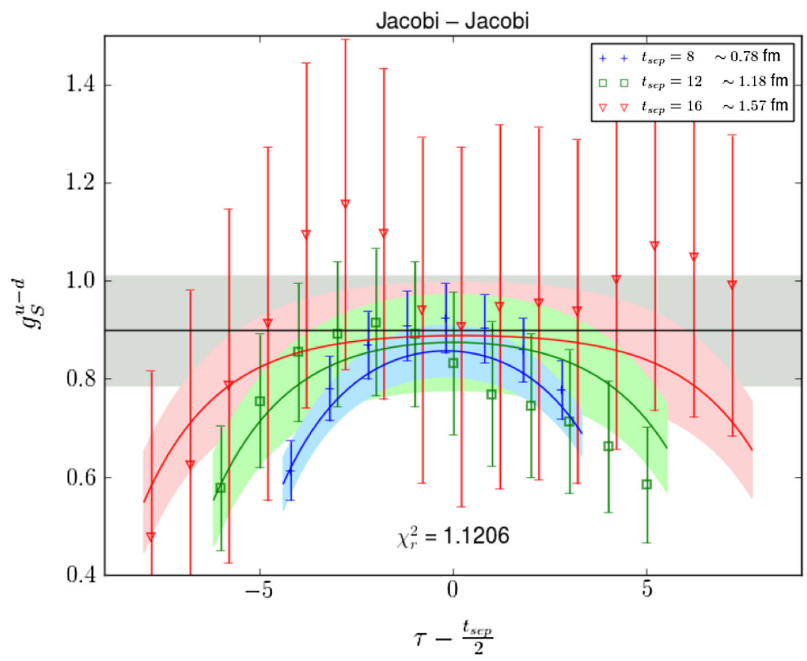

(a)

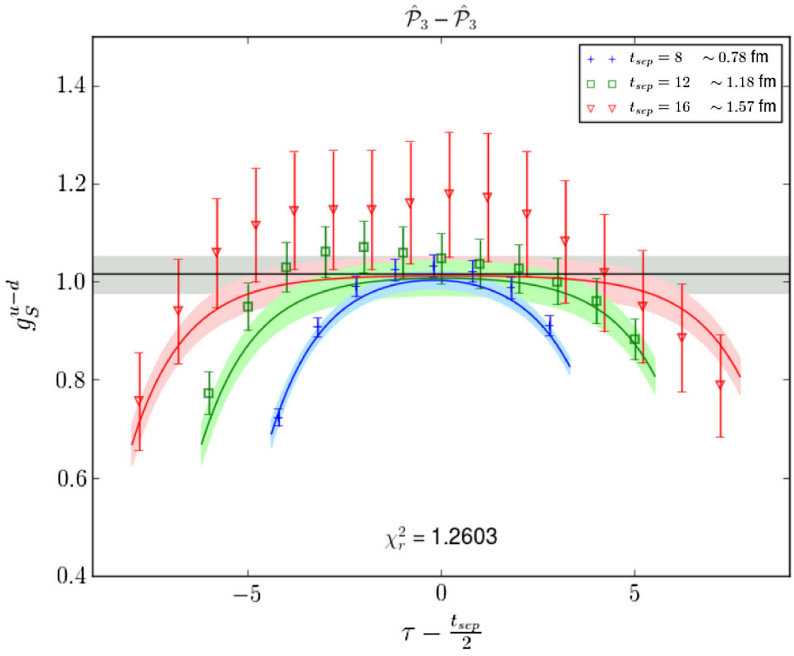

(c) indicating equal excited-state contamination on the source/sink side of the insertion, $\mathcal{M}_{S}^{\text {eff }}$ exhibits antisymmetric behavior for $t_{\mathrm{sep}} / a=12$ and is largely statistical noise for source-sink separations greater than $1.5 \mathrm{fm}$. Immediately noticeable with the use of a single distilled interpolator $\left({ }^{2} S_{S} \frac{1}{2}+\right)$ is the considerable reduction in statistical uncertainty of $\mathcal{M}_{S}^{\text {eff }}$ at each value of $t_{\text {sep }}$. Moreover, the determinations of $\mathcal{M}_{S}^{\text {eff }}$ for different $t_{\text {sep }}$ are in greater agreement, with a clear plateau established for $t_{\text {sep }} \sim 1.18 \mathrm{fm}$. Recalling that a simultaneous fit for all values of $t_{\text {sep }}$ has been performed to extract the matrix element, the fact that the $t_{\text {sep }}=16$ values of $\mathcal{M}_{S}^{\text {eff }}$ are not necessarily on the curve reflects that the fit is largely constrained by data with $t_{\text {sep }} \lesssim 12$. These results are presented in Figs. 2(a) and 2(b).

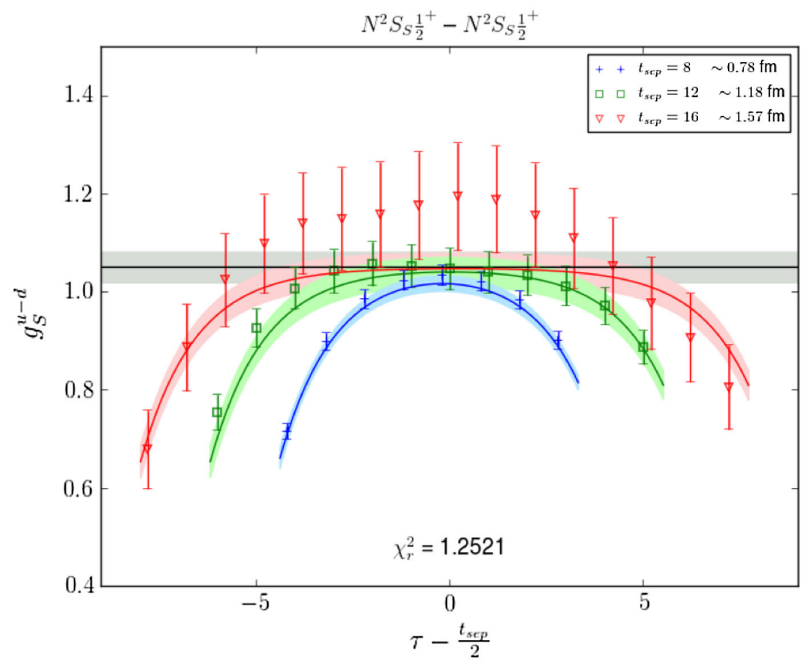

(b)

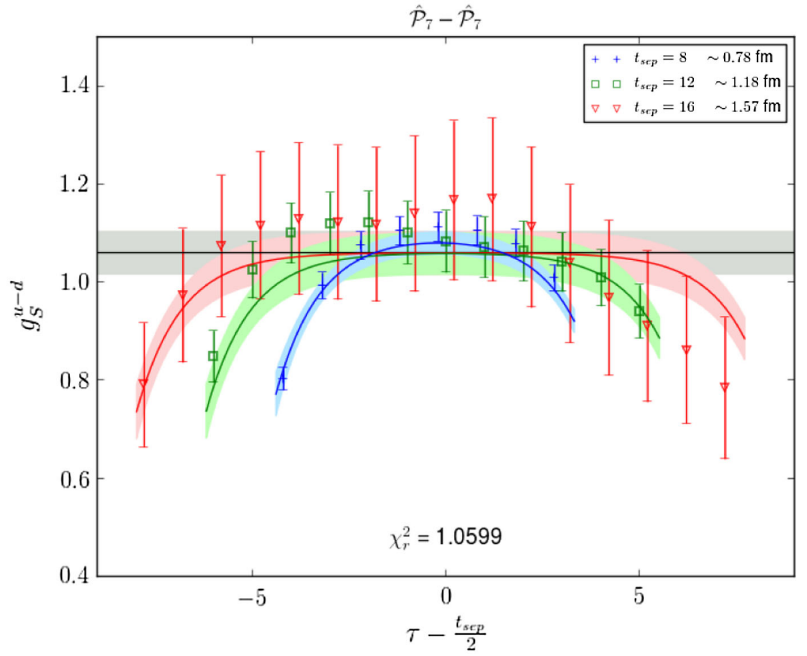

(d)

FIG. 2. Extracted effective unrenormalized isovector scalar charge using the Jacobi-SS (a), ${ }^{2} S_{S^{2}} \frac{1}{2}$ (b), $\hat{\mathcal{P}}_{3}$ (c), and $\hat{\mathcal{P}}_{7}$ (d) interpolators, where 64 eigenvectors were used in the distilled interpolator construction. Displayed plots are for simultaneous fits with $t_{2 \mathrm{pt}}^{\mathrm{fit}} \in[2,16]$ and $\tau_{\text {buff }}=2$. 
Introduction of two hybrid interpolators to construct $\hat{\mathcal{P}}_{3}$ appears to return the $t_{\text {sep }}=8,12$ determinations of $\mathcal{M}_{S}^{\text {eff }}$ to a form structurally similar to that found for the Jacobi-SS interpolator. It is curious to note that the $t_{\text {sep }}=16$ determination of $\mathcal{M}_{S}^{\text {eff }}$ is nearly identical to the corresponding determination with ${ }^{2} S_{S} \frac{1}{2}^{+}$, with inflated errors bars. These data suggest the inclusion of only the two hybrid interpolators provides limited improvement in the extraction of $g_{S}^{u-d}$. Inclusion of the remaining distilled interpolators in the variational analysis yields determinations of $\mathcal{M}_{S}^{\text {eff }}$ that not only exhibit broad plateaus as a function of $\tau$, but are also consistent within error and are consistent with the ${ }^{2} S_{S} \frac{1}{2}^{+}$determination. Results for the scalar insertion with projected interpolators are shown in Figs. 2(c) and 2(d). Simultaneous fit results for each interpolator with scalar current insertion are tabulated in Table III.

\section{2. $g_{A}^{u-d}$}

The isovector axial current $A_{\mu}^{3}=\bar{q} \gamma_{\mu} \gamma_{5} \frac{\tau^{3}}{2} q$ is the insertion that is most sensitive to excited-state contamination and finite-volume effects. As with $g_{S}^{u-d}$, determinations of the effective matrix element (denoted here as $\mathcal{M}_{A}^{\text {eff }}$ ) for different $t_{\text {sep }}$ using the Jacobi-SS interpolator are plagued with poor statistics, as illustrated in Fig. 3. Clearly spatial Gaussian smearing of $\mathcal{N}_{\alpha}$ is not sufficient to suppress excited-state effects.

Employing the local distilled interpolator ${ }^{2} S_{S} \frac{1}{2}$, shown in Fig. 4(b), yet again leads to dramatic reduction in statistical uncertainties in determinations $\mathcal{M}_{A}^{\text {eff }}$, and an increase in the extracted value of $g_{A}^{u-d}$ by $\sim 7 \%$. Notably broad plateaus in $\mathcal{M}_{A}^{\text {eff }}$ can likewise be seen for $t_{\text {sep }}=8,12$ that are consistent

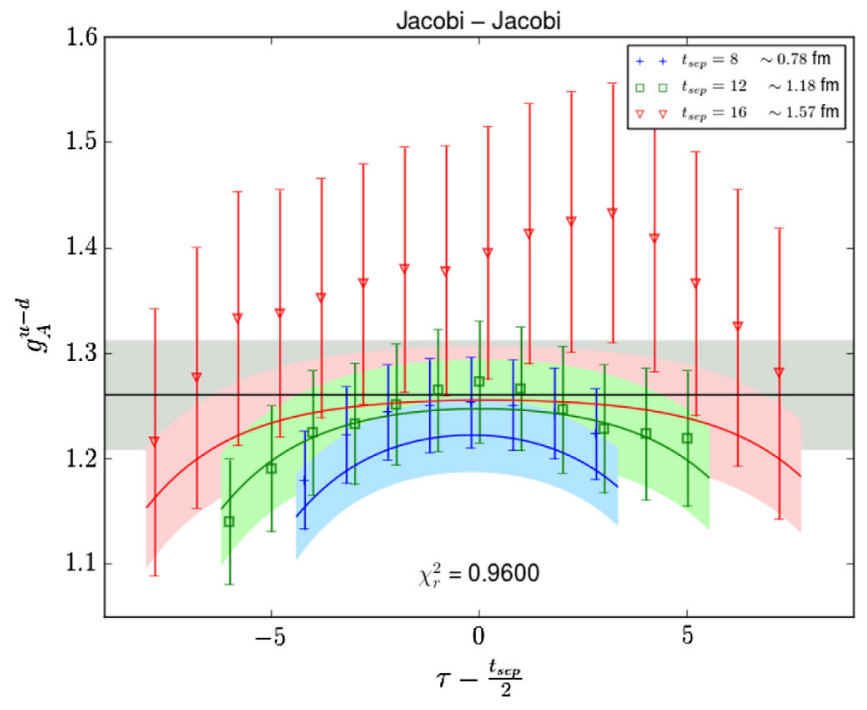

FIG. 3. Extracted effective unrenormalized isovector axial charge using the Jacobi-SS interpolator, where simultaneous fits were performed for $t_{2 \mathrm{pt}}^{\mathrm{fit}} \in[2,16]$ and $\tau_{\text {buff }}=2$. within error, thereby reducing the minimal source-sink separation $(\lesssim 1 \mathrm{fm})$ to reliably extract $g_{A}^{u-d}$.

The behavior of the $\hat{\mathcal{P}}_{3}$ interpolator [Fig. 4(d)] is in line with that found for $g_{S}^{u-d}$-reduced agreement between $t_{\text {sep }}=8,12$ determinations, and fits most heavily constrained by data at smaller source-sink separations. Curiously, the slight oscillation seen in the $t_{\text {sep }}=16$ ${ }^{2} S_{S_{2}}{ }^{1}+$ determination of $\mathcal{M}_{A}^{\text {eff }}$ is amplified following application of the variational method. Prior to $\tau-t_{\text {sep }}<-2$, the $t_{\text {sep }}=16$ effective matrix element seems to fall into agreement with the $t_{\text {sep }}=12$ determination. We speculate the abrupt decrease in the $t_{\text {sep }}=16$ effective matrix element to be caused by neglect of backward-propagating positiveparity states in the corresponding two-point functions.

The $\hat{\mathcal{P}}_{7}$ interpolator perpetuates the double-plateau feature in the $t_{\text {sep }}=16$ determination of $\mathcal{M}_{A}^{\text {eff }}$, shown in Fig. 4(f). Remarkably, however, application of the variational method to this enlarged basis of distilled interpolators demonstrates absolute agreement between the $t_{\text {sep }}=8$, 12 determinations of $\mathcal{M}_{A}^{\text {eff }}$. Simultaneous fit results for each interpolator with axial current insertion are tabulated in Table IV.

\section{3. $g_{T}^{u-d}$}

The isovector tensor current $\mathcal{T}_{\mu \nu}^{3}=\bar{q} \frac{i}{2}\left[\gamma_{\mu}, \gamma_{\nu}\right] \frac{\tau^{3}}{2} q$ is among one of the best determined quantities in the nucleon, particularly at zero virtuality defining $g_{T}^{u-d}$. This facet is supported by models that show excitations of excited states in the nucleon are suppressed when coupling the tensor current $\mathcal{T}_{\mu \nu}^{3}$ [24].

As with previous charges, the ${ }^{2} S_{S} \frac{1}{2}+$ interpolator leads to a dramatic reduction in statistical uncertainty of the effective matrix elements (denoted here as $\mathcal{M}_{T}^{\text {eff }}$ ) when compared to Jacobi-SS, exhibited in Fig. 5(b). By $t_{\text {sep }} \sim 1.18 \mathrm{fm}$, a definite plateau is present in $\mathcal{M}_{T}^{\text {eff }}$ for several insertion times $\tau$; this same plateau is shared with the $t_{\text {sep }}=16$ determination. The absolute agreement of the central values and error of the $t_{\text {sep }}=12,16$ determinations suggest that when using distillation, a source-sink separation of roughly $1 \mathrm{fm}$ is sufficient to reliably extract $g_{T}^{u-d}$.

The variationally improved $\hat{\mathcal{P}}_{3}$ expands upon the improvements seen with the ${ }^{2} S_{S} \frac{1}{2}+$ interpolator. Despite the extracted value of $g_{T}^{u-d}$ being roughly equal [1.133(14) vs $1.147(13)$, respectively] for ${ }^{2} S_{S_{2}}{ }^{+}$and $\hat{\mathcal{P}}_{3}$, the $t_{\text {sep }}=12$, 16 determinations depict an even broader plateau that are again entirely consistent within error. These enlarged plateaus for numerous insertion times $\tau$ follow naturally from a determination of $\Delta m$ that is greater than that determined with ${ }^{2} S_{S} \frac{1}{2}^{+}$. The $\hat{\mathcal{P}}_{7}$ interpolator continues the trends seen with other distilled interpolators, wherein the statistical uncertainties are greatly reduced and the plateaus in the effective matrix elements are seen to become ever larger. Remarkably, the $t_{\text {sep }}=16$ determination using 


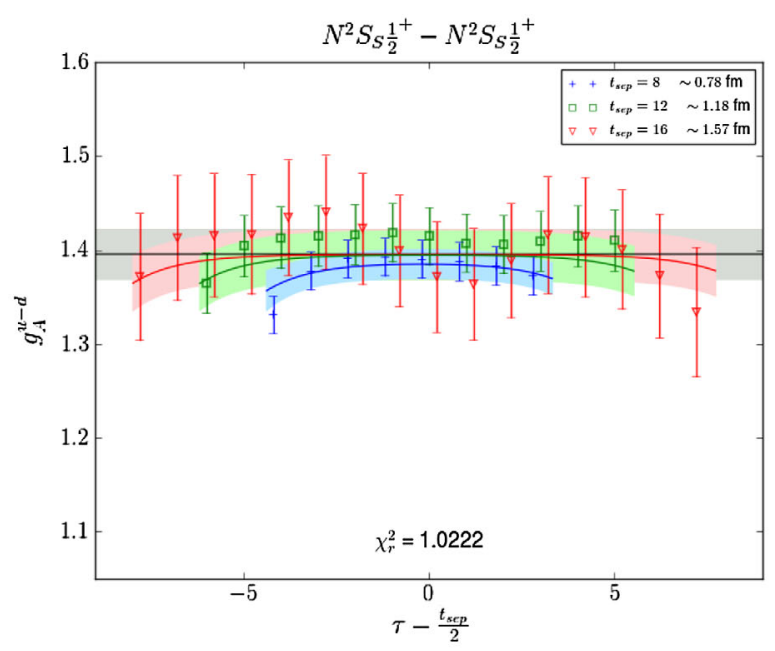

(a)

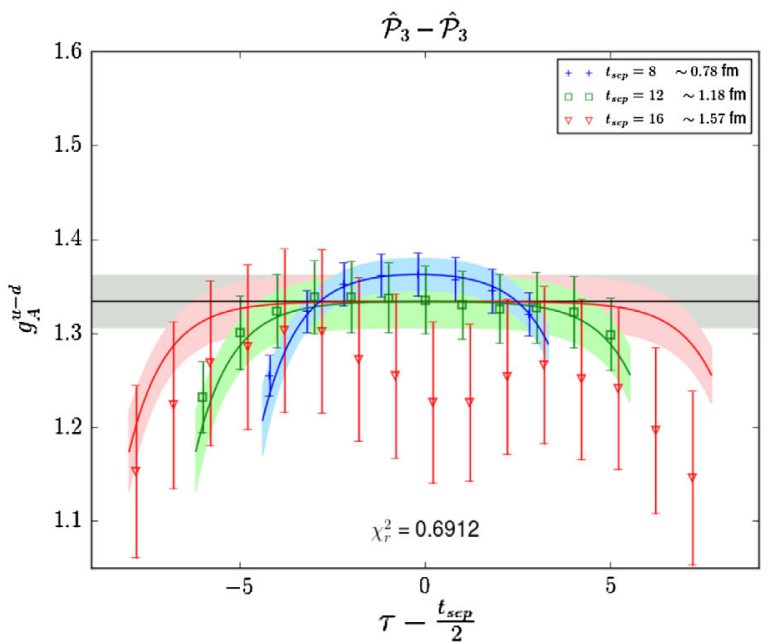

(c)

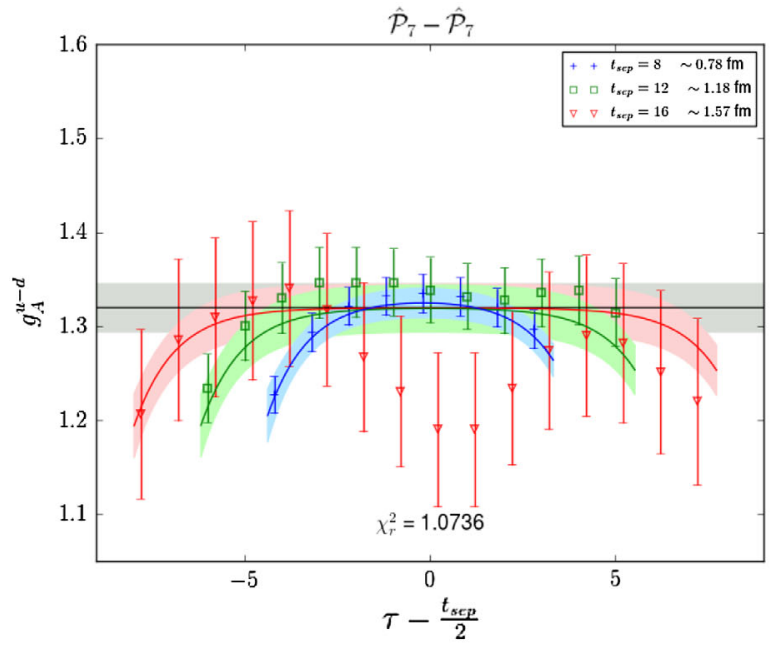

(e)

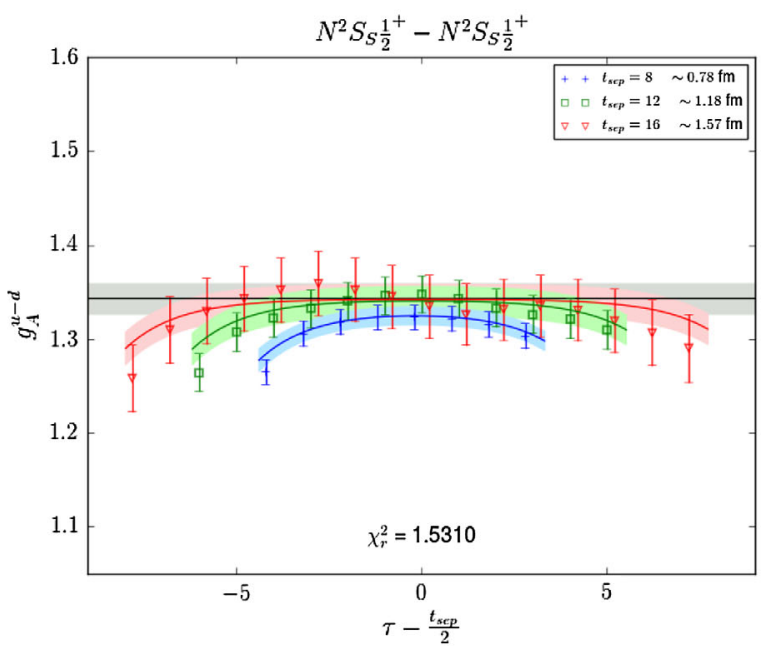

(b)

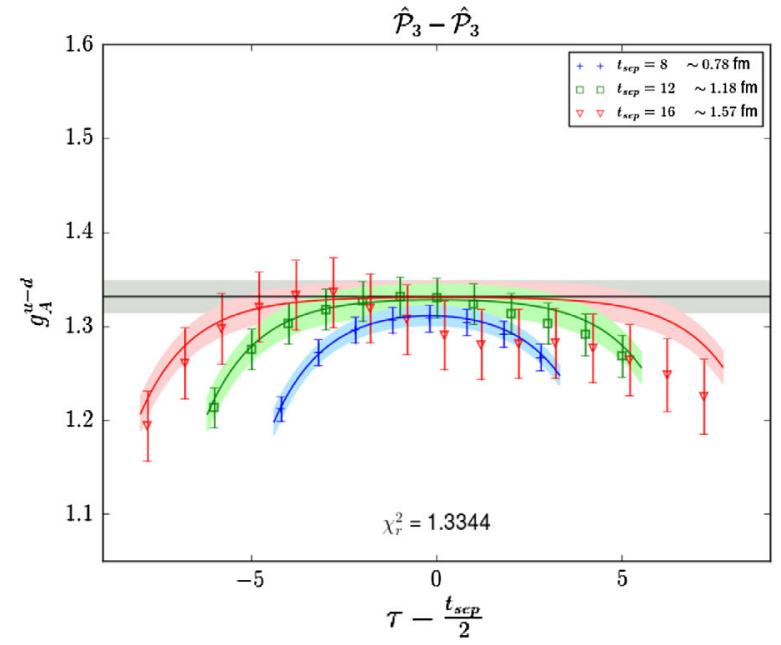

(d)

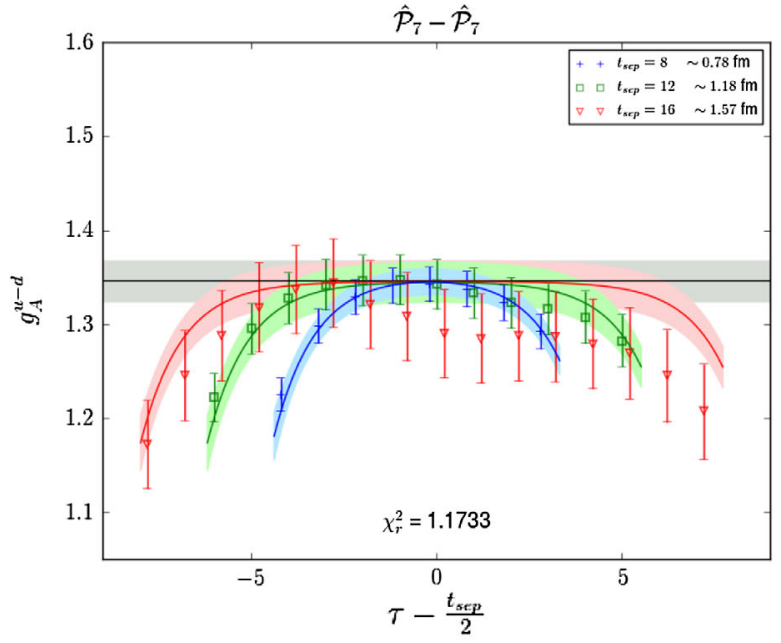

(f)

FIG. 4. Extracted effective unrenormalized isovector axial charge using the ${ }^{2} S_{S_{2}}{ }^{1}$ (upper), $\hat{\mathcal{P}}_{3}$ (middle), and $\hat{\mathcal{P}}_{7}$ (lower) distilled interpolators. Panels (a),(c),(e) correspond to interpolators constructed from 32 eigenvectors, while panels (b),(d),(f) for 64 eigenvectors. Displayed plots are for simultaneous fits with $t_{2 \mathrm{pt}}^{\mathrm{fit}} \in[2,16]$ and $\tau_{\text {buff }}=2$. 


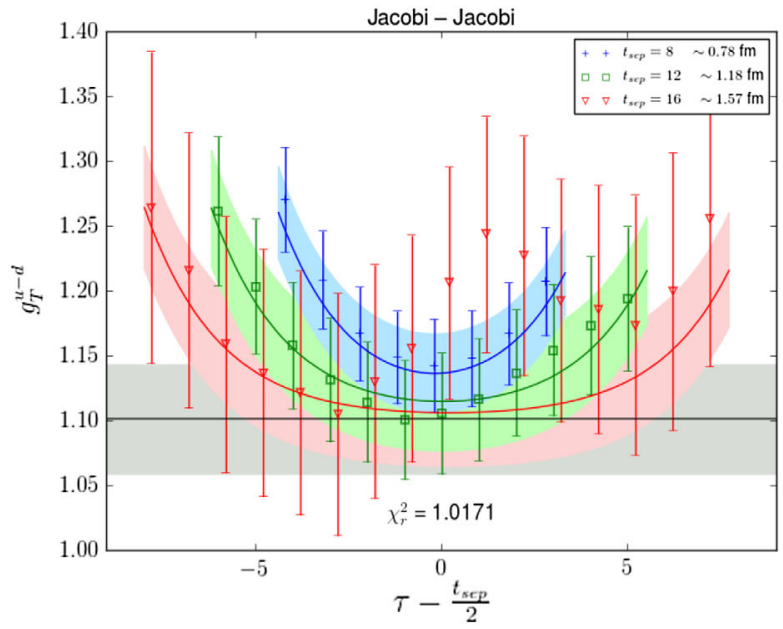

(a)

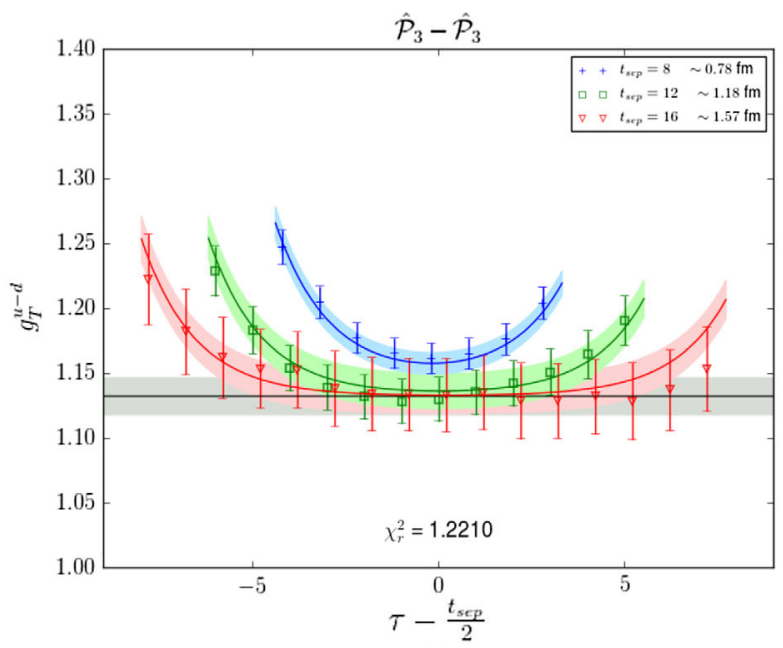

(c)

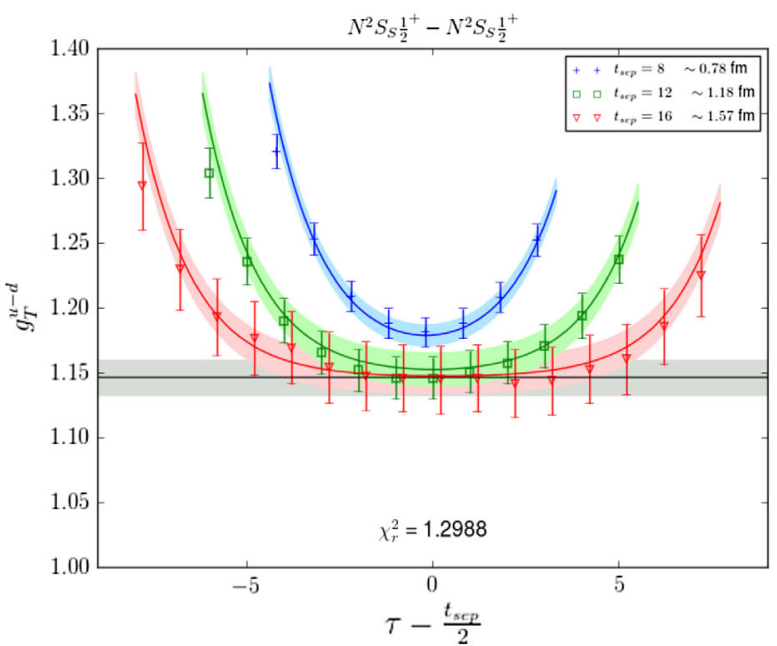

(b)

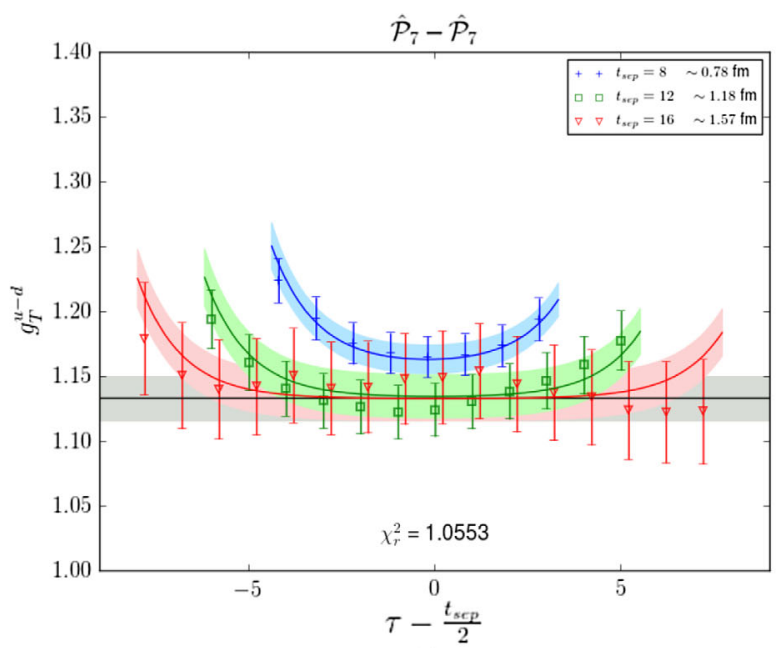

(d)

FIG. 5. Extracted effective unrenormalized isovector tensor charge using the Jacobi-SS (a), ${ }^{2} S_{S}{ }^{1+}$ (b), $\hat{\mathcal{P}}_{3}$ (c), and $\hat{\mathcal{P}}_{7}$ (d) interpolators, where 64 eigenvectors were used in the distilled interpolator construction. Displayed plots are for simultaneous fits with $t_{2 \mathrm{pt}}^{\mathrm{fit}} \in[2,16]$ and $\tau_{\text {buff }}=2$.

$\hat{\mathcal{P}}_{7}$ resembles that of the vector charge-a matrix element that is constant in $\tau$ within minor statistical fluctuations. We emphasize here that the effective matrix element for $\tau-$ $t_{\text {sep }} / 2=-8$ is coincident with the source interpolator and thus should not be considered a reflection of $g_{T}^{u-d}$. Results for the variationally improved interpolators with tensor current insertion are illustrated in Figs. 5(c) and 5(d).

Recalling the functional form of the fit applied to the three-point correlator, Eq. (22), the coefficient $\mathcal{B}$ encodes the first excited-state matrix element and $\mathcal{C}$ captures the ground to first excited-state transition matrix element. As evident from Table $\mathrm{V}$, the determinations of $\mathcal{B}$ for each interpolator are generally consistent with zero, indeed supporting the notion that excited-state contributions to $g_{T}^{u-d}$ are greatly suppressed. On the other hand, the largest source of contamination appears to stem from the transition matrix element contained in $\mathcal{C}$.

$$
\text { 4. } g_{V}^{u-d}
$$

As the isovector vector current $V_{\mu}^{3}=\bar{q} \gamma_{\mu} \frac{\tau^{3}}{2} q$ simply counts the number of quarks within the nucleon, and all its excitations, there is no surprise in the extraction of $g_{V}^{u-d}$ for each interpolator (Fig. 6). We have included the nucleon vector charge here as a useful sanity check, thus ensuring such an observable that is independent of state and $\tau$ is indeed recovered. The continuum requirement $1=Z_{V} g_{V}^{u-d}$ trivially sets the vector current renormalization to be $Z_{V} \sim 0.856$, consistent with determinations on finer lattice ensembles with lighter $m_{\pi}[15,16]$. As for the previous charges, distillation alone affords considerably improved statistics over Jacobi-SS. That said, application of the variational method to a basis of distilled interpolators appears to produce a curious "spreading" in the determined effective vector matrix element. Simultaneous 
TABLE V. Results of simultaneous fits to the two-point and three-point correlators with tensor current insertion. The distilled interpolators were constructed from 64 eigenvectors. The functional forms are given in Eqs. (19) and (22).

\begin{tabular}{|c|c|c|c|c|c|c|c|c|c|}
\hline$\hat{\mathcal{O}}$ & $\tau_{\text {buff }}$ & $\mathcal{A}$ & $\mathcal{B}$ & $\mathcal{C}$ & $M_{0}$ & $M_{1}$ & $|\mathbf{a}|^{2}$ & $|\mathbf{b}|^{2}$ & $g_{T, \text { bare }}^{u-d}$ \\
\hline Jacobi-SS & $\begin{array}{l}1 \\
2 \\
3 \\
4\end{array}$ & $\begin{array}{l}4.70(29) \times 10^{-08} \\
4.49(32) \times 10^{-08} \\
4.48(33) \times 10^{-08} \\
4.50(33) \times 10^{-08}\end{array}$ & $\begin{array}{l}0.21(1.28) \times 10^{-07} \\
3.36(7.57) \times 10^{-08} \\
5.02(7.93) \times 10^{-08} \\
2.45(9.79) \times 10^{-08}\end{array}$ & $\begin{array}{l}1.39(0.11) \times 10^{-08} \\
1.21(0.19) \times 10^{-08} \\
1.31(0.39) \times 10^{-08} \\
2.04(1.28) \times 10^{-08}\end{array}$ & $\begin{array}{l}0.547(5) \\
0.542(6) \\
0.543(6) \\
0.544(6)\end{array}$ & $\begin{array}{l}1.113(71) \\
1.043(73) \\
1.045(74) \\
1.059(79)\end{array}$ & $\begin{array}{l}4.25(19) \times 10^{-08} \\
4.07(23) \times 10^{-08} \\
4.10(23) \times 10^{-08} \\
4.15(23) \times 10^{-08}\end{array}$ & $\begin{array}{l}3.95(28) \times 10^{-08} \\
3.78(23) \times 10^{-08} \\
3.77(24) \times 10^{-08} \\
3.76(26) \times 10^{-08}\end{array}$ & $\begin{array}{l}1.105(41) \\
1.101(42) \\
1.093(43) \\
1.084(45)\end{array}$ \\
\hline${ }^{2} S_{S}{ }^{\frac{1}{2}}{ }^{+}$ & $\begin{array}{l}1 \\
2 \\
3 \\
4\end{array}$ & $\begin{array}{l}1.65(03) \times 10^{-02} \\
1.66(03) \times 10^{-02} \\
1.67(03) \times 10^{-02} \\
1.67(03) \times 10^{-02}\end{array}$ & $\begin{array}{l}4.10(3.26) \times 10^{-02} \\
5.08(3.88) \times 10^{-02} \\
6.03(4.64) \times 10^{-02} \\
3.83(4.94) \times 10^{-02}\end{array}$ & $\begin{array}{l}5.21(0.09) \times 10^{-03} \\
5.51(0.22) \times 10^{-03} \\
6.51(0.53) \times 10^{-03} \\
8.44(1.91) \times 10^{-03}\end{array}$ & $\begin{array}{l}0.534(1) \\
0.535(1) \\
0.536(1) \\
0.536(1)\end{array}$ & $\begin{array}{l}1.204(19) \\
1.225(26) \\
1.245(28) \\
1.246(28)\end{array}$ & $\begin{array}{l}1.44(01) \times 10^{-02} \\
1.45(02) \times 10^{-02} \\
1.46(02) \times 10^{-02} \\
1.46(02) \times 10^{-02}\end{array}$ & $\begin{array}{l}1.61(04) \times 10^{-02} \\
1.64(05) \times 10^{-02} \\
1.68(06) \times 10^{-02} \\
1.68(06) \times 10^{-02}\end{array}$ & $\begin{array}{l}1.146(13) \\
1.147(13) \\
1.147(14) \\
1.144(14)\end{array}$ \\
\hline$\hat{\mathcal{P}}_{3}$ & $\begin{array}{l}1 \\
2 \\
3 \\
4\end{array}$ & $\begin{array}{l}1.20(2) \times 10^{+00} \\
1.19(2) \times 10^{+00} \\
1.20(2) \times 10^{+00} \\
1.19(3) \times 10^{+00}\end{array}$ & $\begin{array}{l}5.40(3.97) \times 10^{+00} \\
4.48(3.28) \times 10^{+00} \\
5.69(4.23) \times 10^{+00} \\
3.63(4.31) \times 10^{+00}\end{array}$ & $\begin{array}{l}2.41(0.06) \times 10^{-01} \\
2.22(0.16) \times 10^{-01} \\
2.91(0.40) \times 10^{-01} \\
5.26(1.67) \times 10^{-01}\end{array}$ & $\begin{array}{l}0.535(1) \\
0.535(1) \\
0.536(2) \\
0.536(2)\end{array}$ & $\begin{array}{l}1.259(34) \\
1.232(40) \\
1.260(45) \\
1.260(45)\end{array}$ & $\begin{array}{l}1.059(11) \times 10^{+00} \\
1.053(13) \times 10^{+00} \\
1.061(13) \times 10^{+00} \\
1.060(13) \times 10^{+00}\end{array}$ & $\begin{array}{l}9.13(37) \times 10^{-01} \\
8.86(40) \times 10^{-01} \\
9.13(47) \times 10^{-01} \\
9.16(48) \times 10^{-01}\end{array}$ & $\begin{array}{l}1.132(14) \\
1.133(14) \\
1.132(14) \\
1.127(15)\end{array}$ \\
\hline$\hat{\mathcal{P}}_{7}$ & $\begin{array}{l}1 \\
2 \\
3 \\
4\end{array}$ & $\begin{array}{l}1.12(2) \times 10^{+00} \\
1.13(3) \times 10^{+00} \\
1.13(3) \times 10^{+00} \\
1.13(3) \times 10^{+00}\end{array}$ & $\begin{array}{l}25.4(15.6) \times 10^{+00} \\
24.2(17.6) \times 10^{+00} \\
32.3(23.9) \times 10^{+00} \\
21.3(21.0) \times 10^{+00}\end{array}$ & $\begin{array}{l}1.62(0.08) \times 10^{-01} \\
1.55(0.23) \times 10^{-01} \\
2.54(0.63) \times 10^{-01} \\
8.60(3.71) \times 10^{-01}\end{array}$ & $\begin{array}{l}0.534(2) \\
0.534(2) \\
0.535(2) \\
0.535(2)\end{array}$ & $\begin{array}{l}1.395(57) \\
1.387(70) \\
1.427(75) \\
1.427(75)\end{array}$ & $\begin{array}{l}9.92(12) \times 10^{-01} \\
9.94(13) \times 10^{-01} \\
9.99(13) \times 10^{-01} \\
9.98(13) \times 10^{-01}\end{array}$ & $\begin{array}{l}1.03(08) \times 10^{+00} \\
1.01(10) \times 10^{+00} \\
1.08(12) \times 10^{+00} \\
1.08(12) \times 10^{+00}\end{array}$ & $\begin{array}{l}1.128(16) \\
1.133(17) \\
1.135(17) \\
1.127(18)\end{array}$ \\
\hline
\end{tabular}

fit results for each interpolator with vector current insertion are tabulated in Table VI.

\section{Numerical cost}

Given the substantial benefits incurred by use of distillation and the variational method in such calculations of hadronic structure, it is worth while to pause and consider the numerical cost of doing so. We highlight that a true oneto-one comparison of distillation to standard techniques is not entirely possible, as distillation is markedly distinct from traditional spatial smearing techniques via sampling of entire time slices.

The calculation of point-to-all propagators in standard lattice calculations proceeds by inverting the chosen discretization of the Dirac operator against a point source in coordinate, spinor, and color space

$$
S(\vec{x}, \vec{z})_{b a}^{\beta \alpha}=\sum_{\vec{y}, \gamma, c} D^{-1}(\vec{x}, \vec{y})_{b c}^{\beta \gamma} \delta(\vec{y}-\vec{z}) \delta_{\gamma \alpha} \delta_{c a}
$$

This operation requires 12 distinct inversions of the Dirac operator, one for each spinor and color index $\{\alpha, a\}$. In our case of the nucleon, with degenerate $u$ and $d$-quarks, this captures the propagation of the nucleon from some source point to all other points on the lattice. Implementing the sequential-source method, as we did for the Jacobi-SS interpolator, reduces the number of required inversions by combining the computed point-to-all propagator with the sink interpolator and using this object as a source for further inversions (deemed sequential propagators). As the $u$-quarks can be combined into one sequential source and the $d$-quarks another, the computation of a three-point function in the sequential-source framework requires two additional inversions against a color and spinor point

TABLE VI. Results of simultaneous fits to the two-point and three-point correlators with vector current insertion. The distilled interpolators were constructed from 64 eigenvectors. The functional forms are given in Eqs. (19) and (22).

\begin{tabular}{|c|c|c|c|c|c|c|c|c|c|}
\hline$\hat{\mathcal{O}}$ & $\tau_{\text {buff }}$ & $\mathcal{A}$ & $\mathcal{B}$ & $\mathcal{C}$ & $M_{0}$ & $M_{1}$ & $|\mathbf{a}|^{2}$ & $|\mathbf{b}|^{2}$ & $g_{V, \text { bare }}^{u-d}$ \\
\hline Jacobi-SS & $\begin{array}{l}1 \\
2 \\
3 \\
4\end{array}$ & $\begin{array}{l}4.68(31) \times 10^{-08} \\
4.70(32) \times 10^{-08} \\
4.71(32) \times 10^{-08} \\
4.97(97) \times 10^{-08}\end{array}$ & $\begin{array}{l}8.30(9.65) \times 10^{-08} \\
8.34(9.55) \times 10^{-08} \\
8.26(9.28) \times 10^{-08} \\
3.19(8.09) \times 10^{-07}\end{array}$ & $\begin{array}{r}-8.62(1.37) \times 10^{-10} \\
-9.62(3.94) \times 10^{-10} \\
0.07(1.57) \times 10^{-09} \\
-0.52(1.75) \times 10^{-07}\end{array}$ & $\begin{array}{l}0.543(6) \\
0.543(6) \\
0.543(6) \\
0.543(6)\end{array}$ & $\begin{array}{l}1.049(77) \\
1.047(77) \\
1.043(77) \\
1.043(76)\end{array}$ & $\begin{array}{l}.13(23) \times 10^{-08} \\
4.13(23) \times 10^{-08} \\
4.13(23) \times 10^{-08} \\
4.12(23) \times 10^{-08}\end{array}$ & $\begin{array}{l}3.73(25) \times 10^{-08} \\
3.71(25) \times 10^{-08} \\
3.69(24) \times 10^{-08} \\
3.70(24) \times 10^{-08}\end{array}$ & $\begin{array}{l}1.131(47) \\
1.137(47) \\
1.140(48) \\
1.21(23)\end{array}$ \\
\hline${ }^{2} S_{S} \frac{1}{2}^{+}$ & $\begin{array}{l}1 \\
2 \\
3 \\
4\end{array}$ & $\begin{array}{l}1.73(03) \times 10^{-02} \\
1.73(03) \times 10^{-02} \\
1.73(03) \times 10^{-02} \\
1.65(05) \times 10^{-02}\end{array}$ & $\begin{array}{r}4.90(5.49) \times 10^{-02} \\
4.82(5.31) \times 10^{-02} \\
4.90(5.31) \times 10^{-02} \\
-4.01(2.63) \times 10^{-01}\end{array}$ & $\begin{array}{r}-1.33(0.13) \times 10^{-04} \\
-8.84(3.77) \times 10^{-05} \\
2.52(1.63) \times 10^{-04} \\
3.99(2.17) \times 10^{-02}\end{array}$ & $\begin{array}{l}0.536(1) \\
0.536(1) \\
0.536(1) \\
0.536(1)\end{array}$ & $\begin{array}{l}1.255(29) \\
1.251(29) \\
1.251(29) \\
1.249(29)\end{array}$ & $\begin{array}{l}1.46(02) \times 10^{-02} \\
1.46(02) \times 10^{-02} \\
1.46(02) \times 10^{-02} \\
1.46(02) \times 10^{-02}\end{array}$ & $\begin{array}{l}1.69(06) \times 10^{-02} \\
1.69(06) \times 10^{-02} \\
1.69(06) \times 10^{-02} \\
1.69(06) \times 10^{-02}\end{array}$ & $\begin{array}{l}1.185(14) \\
1.184(14) \\
1.184(14) \\
1.131(32)\end{array}$ \\
\hline$\hat{\mathcal{P}}_{3}$ & $\begin{array}{l}1 \\
2 \\
3 \\
4\end{array}$ & $\begin{array}{l}1.25(2) \times 10^{+00} \\
1.25(2) \times 10^{+00} \\
1.25(2) \times 10^{+00} \\
1.12(7) \times 10^{+00}\end{array}$ & $\begin{array}{r}5.93(5.05) \times 10^{+00} \\
5.64(4.99) \times 10^{+00} \\
5.41(4.99) \times 10^{+00} \\
-79.5(51.7) \times 10^{+00}\end{array}$ & $\begin{array}{r}-7.16(1.01) \times 10^{-03} \\
-5.78(3.05) \times 10^{-03} \\
1.44(1.39) \times 10^{-02} \\
7.06(3.77) \times 10^{+00}\end{array}$ & $\begin{array}{l}0.535(2) \\
0.535(2) \\
0.536(2) \\
0.535(2)\end{array}$ & $\begin{array}{l}1.265(45) \\
1.262(45) \\
1.265(46) \\
1.261(45)\end{array}$ & $\begin{array}{l}1.061(13) \times 10^{+00} \\
1.061(13) \times 10^{+00} \\
1.061(13) \times 10^{+00} \\
1.058(13) \times 10^{+00}\end{array}$ & $\begin{array}{l}9.21(49) \times 10^{-01} \\
9.19(49) \times 10^{-01} \\
9.21(49) \times 10^{-01} \\
9.22(48) \times 10^{-01}\end{array}$ & $\begin{array}{l}1.179(15) \\
1.179(15) \\
1.181(15) \\
1.056(66)\end{array}$ \\
\hline$\hat{\mathcal{P}}_{7}$ & $\begin{array}{l}1 \\
2 \\
3 \\
4\end{array}$ & $\begin{array}{l}1.18(2) \times 10^{+00} \\
1.18(2) \times 10^{+00} \\
1.18(3) \times 10^{+00} \\
1.07(6) \times 10^{+00}\end{array}$ & $\begin{aligned} 27.9(19.8) & \times 10^{+00} \\
26.0(23.2) & \times 10^{+00} \\
26.1(23.4) & \times 10^{+00} \\
-294(237) & \times 10^{+00}\end{aligned}$ & $\begin{aligned}-1.67(1.16) & \times 10^{-03} \\
0.68(3.75) & \times 10^{-03} \\
1.82(1.94) & \times 10^{-02} \\
13.1(7.1) & \times 10^{+00}\end{aligned}$ & $\begin{array}{l}0.535(2) \\
0.535(2) \\
0.535(2) \\
0.535(2)\end{array}$ & $\begin{array}{l}1.412(70) \\
1.412(75) \\
1.418(75) \\
1.427(77)\end{array}$ & $\begin{array}{l}9.98(13) \times 10^{-01} \\
9.98(13) \times 10^{-01} \\
9.99(13) \times 10^{-01} \\
9.99(13) \times 10^{-01}\end{array}$ & $\begin{array}{l}1.05(11) \times 10^{+00} \\
1.05(11) \times 10^{+00} \\
1.06(11) \times 10^{+00} \\
1.08(12) \times 10^{+00}\end{array}$ & $\begin{array}{l}1.180(17) \\
1.181(18) \\
1.183(18) \\
1.068(53)\end{array}$ \\
\hline
\end{tabular}




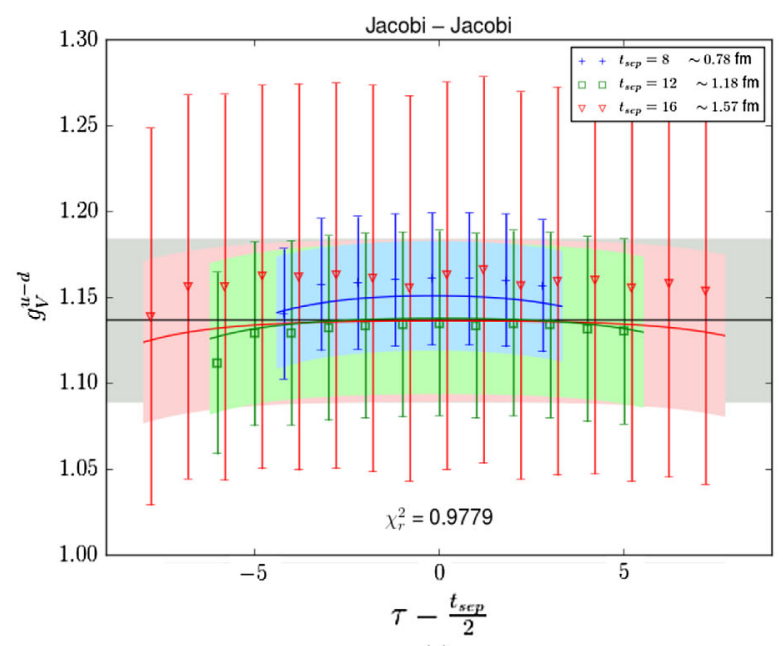

(a)

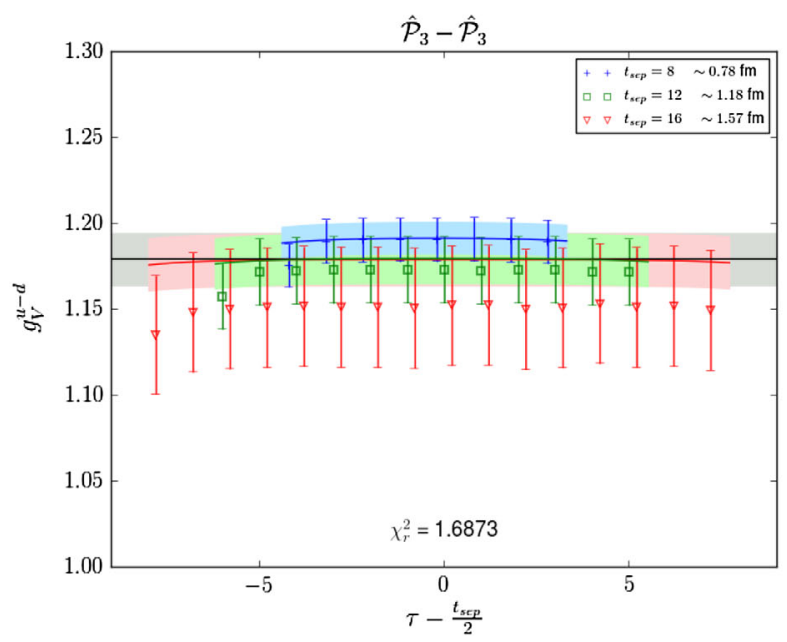

(c)

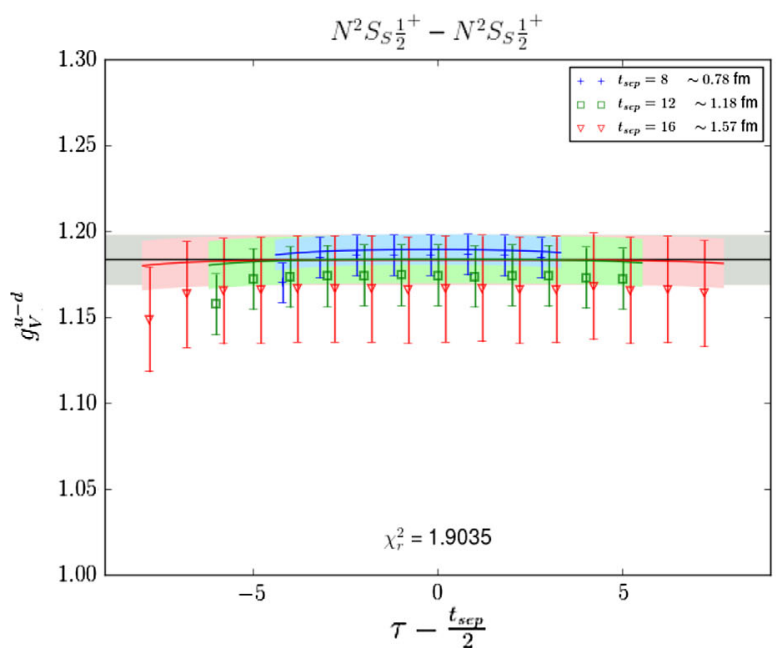

(b)

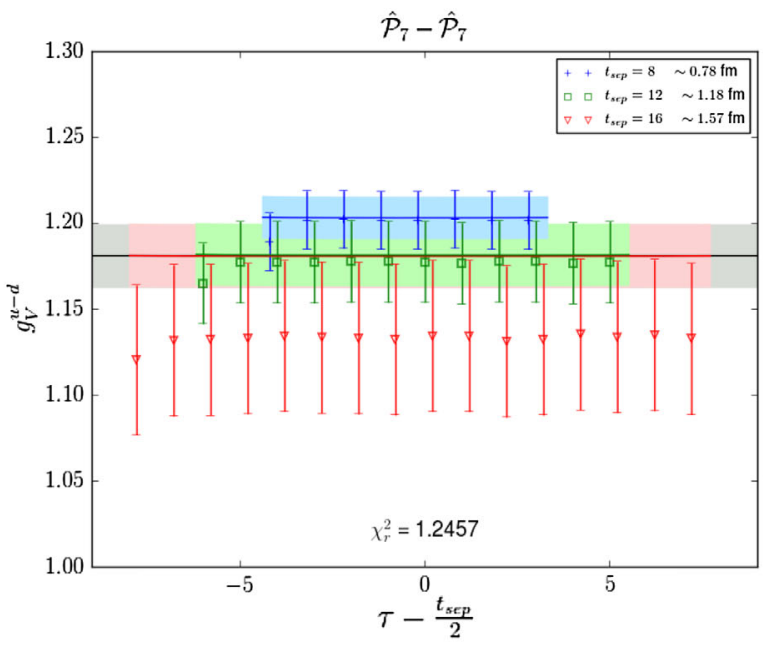

(d)

FIG. 6. Extracted effective unrenormalized isovector vector charge using the Jacobi-SS (a), ${ }^{2} S_{S_{2}}{ }^{1+}$ (b), $\hat{\mathcal{P}}_{3}$ (c), and $\hat{\mathcal{P}}_{7}$ (d) interpolators, where 64 eigenvectors were used in the distilled interpolator construction. Displayed plots are for simultaneous fits with $t_{2 \mathrm{pt}}^{\mathrm{fit}} \in[2,16]$ and $\tau_{\text {buff }}=2$.

source. As the sequential propagators must be recomputed for each new source-sink separation $t_{\text {sep }}$, we arrive at

$$
N_{\text {src }}\left[12+24 \cdot N_{\text {seps }}\right] N_{\text {cfg }}
$$

total inversions of the Dirac operator for a single Jacobismeared interpolator, where $N_{\text {src }}$ is the number of source positions, $N_{\text {seps }}$ the number of source-sink separations, and $N_{\text {cfg }}$ the number of gauge configurations. Were the variational method applied to a two-point correlation matrix of different Jacobi-smeared interpolators, $12 \times N_{\text {ops }}$ inversions would be required to construct the requisite variationally improved interpolator. The total number of inversions in the three-point function remains unchanged, and we would then have

$$
N_{\text {src }}\left[12\left(1+N_{\text {ops }}\right)+24 \cdot N_{\text {seps }}\right] N_{\text {cfg }}
$$

inversions, with $N_{\text {ops }}$ the dimension of the interpolator basis. If instead the variational method was applied to a correlation matrix of different Jacobi-smeared three-point functions, $N_{\mathrm{ops}}$ inversions would be needed at the source and an additional $N_{\text {ops }}$ in the construction of the sequential propagators, for a total

$$
N_{\text {src }} N_{\text {ops }}\left[12+24 \cdot N_{\text {seps }} N_{\text {ops }}\right] N_{\text {cfg }}
$$

where we note that this is now proportional to $N_{\text {ops }}^{2}$.

In the case of distillation, the inversion of the Dirac operator against a point source is replaced with inversion against an eigenvector on some time slice $t$ :

$$
S_{\alpha \beta}^{(k)}\left(\vec{x}, t^{\prime} ; t\right)=D_{\alpha \beta}^{-1}\left(t^{\prime}, t\right) \xi^{(k)}(t) .
$$


As the eigenvectors are determined by solution of $-\nabla^{2}(t) \xi^{(k)}(t)=\lambda^{(k)}(t) \xi^{(k)}(t)$ given some gauge-covariant discretization of $\nabla^{2}$, the calculation of the $k$ th solution vector requires 3 inversions of the Dirac operator, one for each (suppressed) color index. In practice, for a given $t_{\text {sep}}$, we calculate the solution vectors forward (backward) from the source (sink), where the solution vectors from the source are used in the two-point and three-point calculations. Thus for a single distilled interpolator the total number of inversions becomes

$$
3 \cdot N_{\text {src }} N_{\text {eigs }}\left(1+N_{\text {seps }}\right) N_{\text {cfg }}
$$

where $N_{\text {eigs }}$ is the dimension of the distillation space. With $N_{\text {eigs }}=64$ in our case, this cost at first seems excessive. However, once these solution vectors have been computed, any number of interpolating fields, variationally optimized or otherwise, can be correlated without additional cost. We note that we have not taken into account the cost of the Wick contractions when using distillation in this study.

\section{Stability of results with distillation space}

A potential limitation of the standard formulation of Distillation in calculations of hadronic structure is the scaling with spatial volume required to maintain the same resolution of the distillation space. In the case of excitedstate baryon spectroscopy using distillation [2], it was found correlation functions computed on different physical volumes $V_{1}$ and $V_{2}$ had comparable statistical uncertainties and excited-state spectra, provided the number of utilized eigenvectors was adjusted according to $N_{2, \text { vecs }}=\frac{V_{2}}{V_{1}} N_{1, \text { vecs }}$. With the known finite-volume effects present in calculations of the scalar and axial charges of the nucleon, a program to isolate these charges using distillation must necessarily consider the stability of the extracted matrix elements as the volume is increased. As this work is an exploratory study of the differences and advantages of using the variational method and distillation over standard smearing approaches, we maintain the physical volume but repeat the above calculations for the nucleon effective mass and the axial charge where the rank of the distillation space is reduced from 64 to 32 . Reducing the rank of the distillation space is advantageous as it allows the stability of our extracted matrix elements to be explored, while limiting the contraction and inversion costs.

In Fig. 1(a) we display the derived nucleon effective mass using the Jacobi-SS interpolator and distilled interpolators constructed from 32 eigenvectors. It is evident from Figs. 1(a) and 1(b) that reducing the rank of the distillation space has the primary effect of introducing greater statistical uncertainty in the underlying correlation function. However, use of distillation, despite the reduced rank, still leads to an earlier onset of a plateau in the
TABLE VII. Parameters of a single-state fit to the two-point correlators constructed from 32 eigenvectors. Results for $M_{0}$ are consistent with fits applied to the distilled correlators constructed from 64 eigenvectors. Errors are purely statistical.

\begin{tabular}{lcccc}
\hline \hline$\hat{\mathcal{O}}$ & $t_{\text {fit }}$ & $|\mathbf{a}|^{2}$ & $M_{0}$ & $\chi_{r}^{2}$ \\
\hline${ }^{2} S_{S^{2}}{ }^{+}$ & {$[4,16]$} & $2.620(25) \times 10^{-03}$ & $0.548(2)$ & 8.454 \\
& {$[5,16]$} & $2.490(26) \times 10^{-03}$ & $0.541(2)$ & 2.158 \\
& {$[6,16]$} & $2.416(33) \times 10^{-03}$ & $0.538(2)$ & 0.919 \\
& {$[7,16]$} & $2.388(39) \times 10^{-03}$ & $0.537(2)$ & 0.830 \\
$\hat{\mathcal{P}}_{3}$ & {$[4,16]$} & $1.110(11) \times 10^{+00}$ & $0.543(2)$ & 2.675 \\
& {$[5,16]$} & $1.073(13) \times 10^{+00}$ & $0.539(2)$ & 1.021 \\
& {$[6,16]$} & $1.046(17) \times 10^{+00}$ & $0.536(2)$ & 0.445 \\
& {$[7,16]$} & $1.046(21) \times 10^{+00}$ & $0.536(3)$ & 0.501 \\
$\hat{\mathcal{P}}_{7}$ & {$[4,16]$} & $1.101(10) \times 10^{+00}$ & $0.541(2)$ & 2.824 \\
& {$[5,16]$} & $1.068(12) \times 10^{+00}$ & $0.538(2)$ & 0.803 \\
& {$[6,16]$} & $1.051(16) \times 10^{+00}$ & $0.536(2)$ & 0.599 \\
& {$[7,16]$} & $1.045(20) \times 10^{+00}$ & $0.535(2)$ & 0.639 \\
\hline \hline
\end{tabular}

nucleon effective mass when compared to Jacobi-smeared sources, and the limiting behavior of the effective mass is largely unchanged. Results of 1- and 2-state fits applied to the two-point functions with 32 eigenvectors are tabulated in Tables VII and VIII. Of particular importance is that the extracted ground-state mass is stable as the rank of the distillation space is reduced, irrespective of whether a 1- or 2-state fit is applied. The first excited-state energy, however, is 6\%-20\% larger when 32 eigenvectors are used. This difference, although large, is easily understood by recalling that the distillation operator approaches the identity as the number of eigenvectors approaches the number of lattice sites on a time slice. Hence by reducing the number of eigenvectors, the sources comprising our interpolators have a larger extent, and therefore couple even less to high modes.

In Figs. 4(a), 4(c), and 4(e) we plot the effective nucleon matrix element with axial current insertion, for the distilled interpolators constructed from 32 eigenvectors. In the interest of comparison, results with 64 eigenvectors are shown adjacent. As with the nucleon effective mass, reduction in the number of eigenvectors comes at the expense of worsened statistics in the effective matrix element signal. Determinations of $g_{A}^{u-d}$ using the ${ }^{2} S_{S} \frac{1}{2}+$ interpolator appear to be inconsistent as the number of eigenvectors is varied, where $\left.g_{A}^{u-d}\right|_{N_{\text {vec }}=32} \sim 4 \%$ larger than the determination with 64 eigenvectors. These results collectively suggest that statistically significant variations in extracted nucleon charges are possible, were we to repeat these calculations with only a single distilled interpolator on ensembles of larger volumes, unless the rank of the distillation space is increased. On the other hand, there appears to be little dependence of the extracted $g_{A}^{u-d}$ on the number of eigenvectors when distillation is supplemented with the variational method (cf. Table IX). We do mention that methods exist that 
TABLE VIII. Parameters of two-state fits to the two-point correlators constructed from 32 eigenvectors. There is general agreement in determination of the ground-state mass, and agreement is also seen for the same interpolators constructed from 64 eigenvectors (cf. Table II). The first excited-state mass $M_{1}$, however, is noticeably larger when fewer eigenvectors are used-a result entirely consistent with the distillation construction. Errors are purely statistical.

\begin{tabular}{lcrrrrr}
\hline \hline$\hat{\mathcal{O}}$ & \multicolumn{1}{c}{$|\mathbf{a}|^{2}$} & \multicolumn{1}{c}{$M_{0}$} & $|\mathbf{b}|^{2}$ & $M_{1}$ & $\chi_{r}^{2}$ \\
\hline${ }^{2} S_{S^{2}}{ }^{1+}$ & {$[2,16]$} & $2.364(39) \times 10^{-03}$ & $0.537(2)$ & $3.887(239) \times 10^{-03}$ & $1.324(044)$ & 0.602 \\
& {$[3,16]$} & $2.306(174) \times 10^{-03}$ & $0.534(7)$ & $2.82(3.10) \times 10^{-03}$ & $1.184(410)$ & 0.403 \\
& {$[4,16]$} & $2.296(103) \times 10^{-03}$ & $0.534(4)$ & $2.55(1.89) \times 10^{-03}$ & $1.152(254)$ & 0.446 \\
$\hat{\mathcal{P}}_{3}$ & {$[2,16]$} & $1.051(18) \times 10^{+00}$ & $0.537(2)$ & $1.821(237) \times 10^{+00}$ & $1.512(085)$ & 0.622 \\
& {$[3,16]$} & $1.025(30) \times 10^{+00}$ & $0.534(3)$ & $0.948(355) \times 10^{+00}$ & $1.242(174)$ & 0.479 \\
& {$[4,16]$} & $1.028(35) \times 10^{+00}$ & $0.534(3)$ & $1.10(1.39) \times 10^{+00}$ & $1.287(393)$ & 0.531 \\
$\hat{\mathcal{P}}_{7}$ & {$[2,16]$} & $1.042(17) \times 10^{+00}$ & $0.535(2)$ & $1.639(193) \times 10^{+00}$ & $1.459(077)$ & 0.511 \\
& {$[3,16]$} & $1.025(28) \times 10^{+00}$ & $0.534(3)$ & $1.058(439) \times 10^{+00}$ & $1.278(188)$ & 0.468 \\
& {$[4,16]$} & $1.030(45) \times 10^{+00}$ & $0.534(4)$ & $1.38(3.00) \times 10^{+00}$ & $1.356(664)$ & 0.517 \\
\hline \hline
\end{tabular}

TABLE IX. Results of simultaneous fits to the two-point and three-point correlators with axial current insertion. The distilled interpolators were constructed from 32 eigenvectors. The functional forms are given in Eqs. (19) and (22).

\begin{tabular}{|c|c|c|c|c|c|c|c|c|c|}
\hline$\hat{~}$ & 1 & $\mathcal{A}$ & $\mathcal{B}$ & $\mathcal{C}$ & $M_{0}$ & $M_{1}$ & $|\mathbf{a}|^{2}$ & $|\mathbf{b}|^{2}$ & $g_{A, \text { bare }}^{u-d}$ \\
\hline$S_{S} \frac{1}{2}+$ & $\begin{array}{l}1 \\
2 \\
3 \\
4\end{array}$ & $\begin{array}{l}3.335(85) \times 10^{-03} \\
3.322(86) \times 10^{-03} \\
3.345(87) \times 10^{-03} \\
3.307(91) \times 10^{-03}\end{array}$ & $\begin{array}{l}-0.73(3.74) \times 10^{-02} \\
-0.68(3.59) \times 10^{-02} \\
-1.55(3.80) \times 10^{-02} \\
-0.90(3.29) \times 10^{-02}\end{array}$ & $\begin{aligned}-2.19(31) & \times 10^{-04} \\
-1.23(72) & \times 10^{-04} \\
-0.44(1.74) & \times 10^{-04} \\
0.07(8.71) & \times 10^{-04}\end{aligned}$ & $\begin{array}{l}0.538(2) \\
0.538(2) \\
0.538(2) \\
0.537(2)\end{array}$ & $\begin{array}{l}1.349(41) \\
1.343(41) \\
1.349(42) \\
1.327(41)\end{array}$ & $\begin{array}{l}2.382(34) \times 10^{-03} \\
2.379(35) \times 10^{-03} \\
2.385(34) \times 10^{-03} \\
2.365(35) \times 10^{-03}\end{array}$ & $\begin{array}{l}4.00(24) \times 10^{-03} \\
3.96(23) \times 10^{-03} \\
3.98(24) \times 10^{-03} \\
3.90(22) \times 10^{-03}\end{array}$ & $\begin{array}{l}1.400(26) \\
1.396(26) \\
1.403(27) \\
1.398(28)\end{array}$ \\
\hline$\hat{\mathcal{P}}_{3}$ & $\begin{array}{l}1 \\
2 \\
3 \\
4\end{array}$ & $\begin{array}{l}1.407(39) \times 10^{+00} \\
1.415(38) \times 10^{+00} \\
1.427(40) \times 10^{+00} \\
1.417(43) \times 10^{+00}\end{array}$ & $\begin{array}{l}105.9(103.1) \\
96.5(100.3)\end{array}$ & $\begin{array}{r}-2.33(23) \times 10^{-01} \\
-2.76(66) \times 10^{-01} \\
-2.94(1.76) \times 10^{-01} \\
-0.22(1.07) \times 10^{+00}\end{array}$ & $\begin{array}{l}0.538(2) \\
0.538(2) \\
0.538(2) \\
0.538(2)\end{array}$ & $\begin{array}{l}1.554(83) \\
1.558(75) \\
1.563(76) \\
1.537(77)\end{array}$ & $\begin{array}{l}1.059(16) \times 10^{+00} \\
1.060(15) \times 10^{+00} \\
1.061(15) \times 10^{+00} \\
1.057(16) \times 10^{+00}\end{array}$ & $\begin{array}{l}1.944(255) \times 10^{+00} \\
1.966(231) \times 10^{+00} \\
1.972(235) \times 10^{+00} \\
1.891(225) \times 10^{+00}\end{array}$ & $\begin{array}{l}1.328(33) \\
1.335(28) \\
1.345(28) \\
1.341(31)\end{array}$ \\
\hline$P_{7}$ & $\begin{array}{l}1 \\
2 \\
3 \\
4\end{array}$ & $\begin{array}{l}1.373(38) \times 10^{+00} \\
1.379(37) \times 10^{+00} \\
1.378(37) \times 10^{+00} \\
1.382(42) \times 10^{+00}\end{array}$ & $17.64(49.75)$ & $\begin{aligned}-2.13(20) & \times 10^{-01} \\
-2.18(50) & \times 10^{-01} \\
-1.67(1.25) & \times 10^{-01} \\
0.13(7.71) & \times 10^{-01}\end{aligned}$ & $\begin{array}{l}0.536(2) \\
0.536(2) \\
0.536(2) \\
0.536(2)\end{array}$ & $\begin{array}{l}1.443(66) \\
1.447(68) \\
1.450(68) \\
1.463(71)\end{array}$ & $\begin{array}{l}1.044(15) \times 10^{+00} \\
1.044(16) \times 10^{+00} \\
1.044(16) \times 10^{+00} \\
1.044(16) \times 10^{+00}\end{array}$ & $\begin{array}{l}1.604(161) \times 10^{+00} \\
1.614(167) \times 10^{+00} \\
1.622(169) \times 10^{+00} \\
1.657(179) \times 10^{+00}\end{array}$ & $\begin{array}{l}1.315(28) \\
1.321(26) \\
1.319(25) \\
1.324(31)\end{array}$ \\
\hline
\end{tabular}

circumvent the poor volume scaling of distillation. One such method includes the Laplacian-Heaviside [2] (LapH) smearing method, where distillation is supplemented with a hard cutoff via

$$
\square(t)_{\mathrm{LapH}}=\sum_{k=1}^{N} \Theta\left(\lambda-\lambda_{k}\right) \xi^{(k)}(t) \xi^{(k) \dagger}(t)
$$

where $\lambda$ imposes the cutoff for all eigenvalues $\lambda_{k}>\lambda$. In this manner, only the desired eigenvectors are used in construction of elementals, solution vectors, and perambulators. The density of eigenmodes still scales linearly with the volume, however, a situation amenable to stochastic estimation $[25,26]$. As our program for extracting nucleon matrix elements relies on exact determination of the distillation space, the need for the variational method on larger volumes cannot be overstated.

\section{CONCLUSIONS}

We have investigated the use of distillation, and an extended basis of interpolators, in the calculation of the scalar, axial and tensor isovector charges of the nucleon, and made comparisons with a calculation on the same ensemble using the conventional Jacobi-smearing method of a single smearing radius. We find that distillation affords a considerable improvement in the statistical quality of the data when compared with calculations using Jacobi smearing. We attribute this improvement to be a consequence of momentum projection performed at both source and sink in the case of a two-point function, and at source, sink and current in the case of a three-point function. More surprisingly, even the use of a single, local distilled interpolating operator results in a suppression of the contribution of excited states relative to that of the ground state in both two-point and three-point functions.

For our variational analysis, we employed a basis of operators comprising the nonrelativistic operators that 
can be constructed with up to two covariant derivatives, together with so-called "hybrid" operators where the gluons play a manifest role. Whilst the improvement was not as dramatic as that between a single Jacobi-smeared and a single distillation-smeared operator, the use of the variational method and the extended basis provided more consistency and fidelity in the matrix elements for different source-sink separations. Furthermore, the extended basis can be introduced without further propagator calculations, in contrast to the case of Jacobi smearing where the use of the variational method demands a considerable increase in the number of propagators to be computed.

Due to the scaling of distillation with the lattice spatial volume, we further investigated the dependence of our results on the number of utilized eigenvectors. A nontrivial change in the extracted axial charge was observed when using the local distilled interpolator with half as many eigenvectors, while no statistically meaningful difference was observed in the axial charge when our variationally optimized interpolators were employed. The same behavior was noticed with the scalar, tensor and vector charges, but was omitted for brevity. A future study will explore the possibility of using the variational method to sidestep the costly scaling of distillation in calculations of nucleon matrix elements on larger volumes.

The next step in our program is to extend our investigation from matrix elements between nucleons at rest to those in motion, and from forward matrix elements to offforward matrix elements. The former is key to the efficient application of the quasi-PDF, pseudo-PDF and currentcurrent correlator methods to the calculation of parton distribution functions in the nucleon, while the latter is important for form factors at high momenta, and off-forward matrix elements such as generalized parton distributions. Nonetheless, the work presented here clearly demonstrates the efficacy of distillation as a means both of decreasing the statistical uncertainty, and of reducing excited-state contributions, in calculations of nucleon properties.

\section{ACKNOWLEDGMENTS}

Calculations were performed using the Chroma [27] software suite on the computing clusters at Jefferson Lab. We are grateful to Jo Dudek and Stefan Meinel for the use of their fitting codes, and to Robert Edwards for invaluable discussions on the feasibility of these calculations. C. E. extends thanks to Balint Joó for invaluable discussions on building software on the varied machine architectures on the Jefferson Lab clusters. This material is based upon work supported by the U.S. Department of Energy, Office of Science, Office of Nuclear Physics under Contract No. DE-AC05-06OR23177. Computations for this work were carried out in part on facilities of the USQCD Collaboration, which are funded by the Office of Science of the U.S. Department of Energy. C. E. was supported in part by the U.S. Department of Energy under Contract No. DE-FG02-04ER41302 and a Department of Energy Office of Science Graduate Student Research fellowship, through the U.S. Department of Energy, Office of Science, Office of Workforce Development for Teachers and Scientists, Office of Science Graduate Student Research (SCGSR) program. The SCGSR program is administered by the Oak Ridge Institute for Science and Education (ORISE) for the DOE. ORISE is managed by ORAU under Contract No. DE-SC0014664.
[1] C. C. Chang et al., Nature (London) 558, 91 (2018).

[2] M. Peardon, J. Bulava, J. Foley, C. Morningstar, J. Dudek, R. G. Edwards, B. Joo, H.-W. Lin, D. G. Richards, and K. J. Juge (Hadron Spectrum Collaboration), Phys. Rev. D 80, 054506 (2009).

[3] M. Tanabashi et al. (Particle Data Group), Phys. Rev. D 98, 030001 (2018).

[4] H.-W. Lin, R. Gupta, B. Yoon, Y.-C. Jang, and T. Bhattacharya, Phys. Rev. D 98, 094512 (2018).

[5] S. Ohta (RBC, UKQCD, LHP Collaborations), Proc. Sci. LATTICE2015 (2016) 124.

[6] N. Yamanaka, S. Hashimoto, T. Kaneko, and H. Ohki (JLQCD Collaboration), Phys. Rev. D 98, 054516 (2018).

[7] J. Liang, Y.-B. Yang, K.-F. Liu, A. Alexandru, T. Draper, and R. S. Sufian, Phys. Rev. D 96, 034519 (2017).

[8] Y.-B. Yang, A. Alexandru, T. Draper, M. Gong, and K.-F. Liu, Phys. Rev. D 93, 034503 (2016).
[9] R. Gupta, Y.-C. Jang, B. Yoon, H.-W. Lin, V. Cirigliano, and T. Bhattacharya, Phys. Rev. D 98, 034503 (2018).

[10] R. Gupta, T. Bhattacharya, Y.-C. Jang, H.-W. Lin, and B. Yoon, EPJ Web Conf. 175, 06029 (2018).

[11] C. Alexandrou et al., Phys. Rev. D 95, 114514 (2017); 96, 099906(E) (2017).

[12] C. Alexandrou, M. Constantinou, K. Hadjiyiannakou, K. Jansen, C. Kallidonis, G. Koutsou, and A. Vaquero AvilesCasco, Phys. Rev. D 96, 054507 (2017).

[13] G. S. Bali, S. Collins, B. Glässle, M. Göckeler, J. Najjar, R. H. Rödl, A. Schäfer, R. W. Schiel, W. Søldner, and A. Sternbeck, Phys. Rev. D 91, 054501 (2015).

[14] R. Horsley, Y. Nakamura, A. Nobile, P. E. L. Rakow, G. Schierholz, and J. M. Zanotti, Phys. Lett. B 732, 41 (2014).

[15] B. Yoon et al., Phys. Rev. D 93, 114506 (2016).

[16] B. Yoon et al., Phys. Rev. D 95, 074508 (2017). 
[17] B. J. Owen, J. Dragos, W. Kamleh, D. B. Leinweber, M. S. Mahbub, B. J. Menadue, and J. M. Zanotti, Phys. Lett. B 723, 217 (2013).

[18] C. R. Allton et al. (UKQCD Collaboration), Phys. Rev. D 47, 5128 (1993).

[19] R. G. Edwards, J. J. Dudek, D. G. Richards, and S. J. Wallace, Phys. Rev. D 84, 074508 (2011).

[20] J. J. Dudek and R. G. Edwards, Phys. Rev. D 85, 054016 (2012).

[21] J. J. Dudek, R. G. Edwards, N. Mathur, and D. G. Richards, Phys. Rev. D 77, 034501 (2008).

[22] C. Morningstar and M. J. Peardon, Phys. Rev. D 69, 054501 (2004).
[23] B. Blossier, M. Della Morte, G. von Hippel, T. Mendes, and R. Sommer, J. High Energy Phys. 04 (2009) 094.

[24] C. Roberts (private communication).

[25] C. Morningstar, A. Bell, J. Bulava, E. Engelson, J. Foley, K. J. Juge, D. Lenkner, M. Peardon, S. Wallace, and C. H. Wong, AIP Conf. Proc. 1257, 779 (2010).

[26] J. Bulava, J. Foley, Y. C. Jhang, K. J. Juge, D. Lenkner, M. J. Peardon, C. J. Morningstar, and C.H. Wong, arXiv: 1109.6614.

[27] R. G. Edwards and B. Joo (SciDAC, LHPC, UKQCD Collaborations), Nucl. Phys. B, Proc. Suppl. 140, 832 (2005). 